June 2004

CERN-TH/2004-100

\title{
Split Supersymmetry
}

\author{
G. F. Giudice and A. Romanino \\ CERN, Theory Division, CH-1211 Geneva 23, Switzerland
}

\begin{abstract}
The naturalness criterion applied to the cosmological constant implies a new-physics threshold at $10^{-3} \mathrm{eV}$. Either the naturalness criterion fails, or this threshold does not influence particle dynamics at higher energies. It has been suggested that the Higgs naturalness problem may follow the same fate. We investigate this possibility and, abandoning the hierarchy problem, we use unification and dark matter as the only guiding principles. The model recently proposed by Arkani-Hamed and Dimopoulos emerges as a very interesting option. We study it in detail, analysing its structure, and the conditions for obtaining unification and dark matter.
\end{abstract}




\section{Introduction}

For decades the naturalness (or hierarchy) problem of the Higgs mass term has been the guiding principle to construct theories beyond the Standard Model (SM). The criterion of naturalness has the exciting implication that the SM should stop to be valid at a scale around the $\mathrm{TeV}$, and new dynamics should occur at energies reachable by present or nearfuture colliders. Although no clear indications for any SM failure at electroweak energies has emerged so far, a conclusive resolution of this issue has to wait for the LHC.

From a field-theoretical point of view, the cosmological constant problem appears to be very similar to the naturalness problem of the Higgs mass, since both of them are related to ultraviolet power divergences. The same naturalness criterion, applied to the cosmological costant, leads to the existence of a threshold of new dynamics at $10^{-3} \mathrm{eV}$. We do not know if some hidden dynamics actually takes place at that scale, or if the resolution of the problem comes without any modification of the dynamics. What we know is that present particle physics calculations, valid at energies much larger than $10^{-3} \mathrm{eV}$, can be safely performed by setting the cosmological constant to zero and ignore any effect caused by the mechanism ultimately responsible for the solution of the problem. This fact has been justified by invoking the anthropic principle [1, which could be operating in presence of a large number of metastable vacua, as in string theory [2].

It is conceivable to ponder whether such an explanation could also apply to the hierarchy problem, imagining a mechanism (not necessarily based on the anthropic principle) which allows to extrapolate SM calculations to energies much larger than the TeV, without the need of introducing new dynamics, besides the Higgs.

At first sight, this sounds like a devastating proposal. But, if we are willing to abandon the hierarchy problem, we can try to use other clues to drive the search for the theory beyond the SM. Gauge coupling unification could be one such clue: it is motivated by a theory that addresses questions related to the fundamental structure of the SM particle content.

The failure of exact unification of gauge couplings in the SM suggests the existence of new particles, belonging to incomplete GUT irreps, which mend the mismatch. It is well known that low-energy supersymmetry provides precisely the necessary particles with the appropriate quantum numbers. Recently, Arkani-Hamed and Dimopoulos [3, setting aside the hierarchy problem, have noticed that gauge-coupling unification can be achieved in a supersymmetric model where all scalars, but one Higgs doublet, are much heavier than the electroweak scale. Most of the unpleasent aspects of supersymmetry (excessive flavour 
and CP violation, fast dimension-5 proton decay, tight constraints on the Higgs mass) are eliminated, but the unification is retained.

If supersymmetry plays no rôle in solving the hierarchy problem, there is no reason to insist that the spectrum is (partially) supersymmetric. Therefore, in this paper we perform a general analysis of the particle content that has to be added to the SM to obtain gaugecoupling unification. We assume that the new particles have masses around the weak scale, and postulate the absence of thresholds at intermediate energies or various stages of gauge symmetry breaking. Why should the new states appear at the weak scale, if the hierarchy problem is not the guiding principle of the analysis?

Here we can use a second observational clue: the evidence for dark matter and the observation that a particle with weak cross section and mass around the Fermi scale is a natural candidate for it. We stress that the unification and dark-matter arguments are not in general sufficient to insure that new physics be within the LHC discovery reach, contrary to the naturalness criterion. Nevertheless, as we will show in our analysis, in some cases there are interesting experimental consequences to be investigated.

In particular, as explained in sect. 2.1] we find that the model proposed in ref. [3], which we call Split Supersymmetry, emerges as more justified than other non-supersymmetric scenarios. For this reason, in sect. 3. we perform a careful analysis of the predictions of Split Supersymmetry, extending the results of ref. 3. We perform a two-loop analysis of gauge coupling unification and discuss the $m_{b} / m_{\tau}$ relation. We discuss the predictions for gaugino masses and couplings and for the Higgs mass. Next, we link the gaugino and higgsino masses to the weak scale by computing the relic abundance of the lightest neutralino and by requiring that it constitutes the dark matter. Finally, we briefly discuss signals at high-energy colliders.

\section{Conditions for Gauge-Coupling Unification}

In this section we want to classify all possible particle contents that lead to a successful gaugecoupling unification, under the two following assumptions: (i) grand unification into a simple group occurs with no intermediate stages of symmetry breaking; (ii) the new particles have masses comparable with the electroweak scale. We do not necessarily assume low-energy supersymmetry.

Considering one-loop evolution of gauge couplings, the GUT predictions of the strong 
coupling $\alpha_{s}$, and of the unification scale $M_{\mathrm{GUT}}$ and coupling $\alpha_{\mathrm{GUT}}$ are

$$
\begin{aligned}
\alpha_{s}^{-1} & =\alpha^{-1}\left[\sin ^{2} \theta_{W}+\frac{3-8 \sin ^{2} \theta_{W}}{5}\left(\frac{b_{3}-b_{2}}{b_{1}-b_{2}}\right)\right], \\
\ln \frac{M_{\mathrm{GUT}}}{M_{Z}} & =\frac{2 \pi\left(3-8 \sin ^{2} \theta_{W}\right)}{5 \alpha\left(b_{1}-b_{2}\right)}, \\
\alpha_{\mathrm{GUT}} & =\frac{5 \alpha\left(b_{1}-b_{2}\right)}{5 \sin ^{2} \theta_{W} b_{1}-3 \cos ^{2} \theta_{W} b_{2}}, \\
b_{3} & =\frac{1}{3}\left(4 N_{g}-33+N_{3}\right) \\
b_{2} & =\frac{1}{3}\left(4 N_{g}-22+\frac{n_{H}}{2}+N_{2}\right), \\
b_{1} & =\frac{1}{3}\left(4 N_{g}+\frac{3 n_{H}}{10}+N_{1}\right) .
\end{aligned}
$$

Here $N_{g}$ counts the contribution to the $\beta$-functions from complete $S U(5)$ irreps, and it is normalized such that the 3 families of SM quarks and leptons give $N_{g}=3$. In the case of low-energy supersymmetry $N_{g}=9 / 2$, because of the extra contributions from squarks and sleptons (which count $1 / 2$ for each generation). However, notice that $N_{g}$ does not affect the predictions of $\alpha_{s}$ and $M_{\mathrm{GUT}}$, since its contribution cancels in the difference of two $\beta$-function coefficients. Next, $n_{H}$ counts the number of Higgs doublets, and we take $n_{H}=1$. Finally, $N_{i}$ give the contributions from matter in incomplete GUT multiplets. The normalization is such that, for two-component fermions, $N_{i}$ is the Dynkin index of the irrep ${ }^{1}$ and, for complex scalars, $N_{i}$ is half the Dynkin index. With this definition, all $N_{i}$ are positive, and $N_{2,3}$ are integers for chiral fermions, even integers for fermions in real irreps, and half integers for complex scalars. The same holds for the quantity $5 N_{1}$, if the representation can be embedded in $S U(5) .^{2}$

\footnotetext{
${ }^{1}$ In the case of $S U(N)$ groups, the Dynkin index is 1 for the fundamental, $2 N$ for the adjoint, and $N+2$ for the symmetric product of two fundamentals. In the case of $U(1)_{Y}$, we define the Dynkin index as $(3 / 10) N_{f} Y^{2}$, where $N_{f}$ is the number of multiplet components and $Y$ is the hypercharge $\left(Y^{2}=1\right.$ for the SM Higgs).

${ }^{2}$ This can be proven by using the following observation. Let us consider an irrep of $S U(N)$ in a subspace $\Sigma$ of a tensor product of fundamentals. Every linear operator $X$ acting on the space of the fundamental representation induces a linear operator $X_{\Sigma}$ in $\Sigma$, defined as the sum of the action of $X$ on the single tensor factors. We then have
}

$$
\begin{gathered}
\operatorname{Tr} X_{\Sigma}=r_{\Sigma} \operatorname{Tr} X \\
\operatorname{Tr} X_{\Sigma} Y_{\Sigma}=p_{\Sigma} \operatorname{Tr} X \operatorname{Tr} Y+q_{\Sigma} \operatorname{Tr} X Y
\end{gathered}
$$

where $p_{\Sigma}, q_{\Sigma}, r_{\Sigma}$ are non-negative integers independent of $X, Y$. Let us now consider a SM chiral fermion irrep from $S U(5)$. That can be written as the tensor product of an irrep of $S U(2)$ with dimension $D_{2}$ and an irrep of $S U(3)$ with dimension $D_{3}$. Moreover, the hypercharge gives 1 on each $S U(2)$ factor and $-2 / 3$ on 


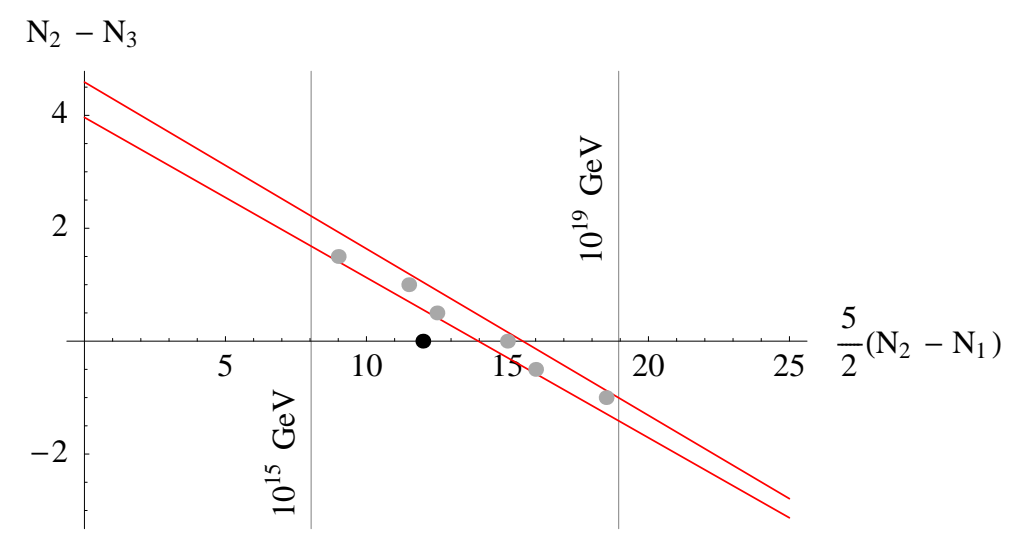

Figure 1: On the plane of the Dynkin indices of new matter $\left(N_{2}-N_{3}\right)$ and $\left(N_{2}-N_{1}\right) 5 / 2$, the diagonal band shows the region where $\alpha_{s}\left(M_{Z}\right)$ is within $2-\sigma$ of its measured value. Points along the vertical lines give the same value of $M_{\mathrm{GUT}}$, and the lines corresponding to $M_{\mathrm{GUT}}=10^{15} \mathrm{GeV}$ and $10^{19} \mathrm{GeV}$ are shown. The black dot indicates the solution with only fermions in real irreps and the gray dots indicate solutions when also new scalar particles are added.

In fig. 1 we show the predictions for $\alpha_{s}\left(M_{Z}\right)$ and $M_{\mathrm{GUT}}$ as functions of $N_{2}-N_{3}$ and $\left(N_{2}-N_{1}\right) 5 / 2$, taking $\sin ^{2} \theta_{W}\left(M_{Z}\right)=0.23150 \pm 0.00016$ and $\alpha^{-1}\left(M_{Z}\right)=128.936 \pm 0.0049$ [4]. Agreement with the measured value $\alpha_{s}\left(M_{Z}\right)=0.119 \pm 0.003$ gives a well-defined correlation between $N_{2}-N_{3}$ and $\left(N_{2}-N_{1}\right) 5 / 2$, shown by the diagonal band in fig. 11. The requirement that unification is achieved $\left(\alpha_{\mathrm{GUT}}>0\right)$ in the perturbative domain $\left(\alpha_{\mathrm{GUT}}<1\right)$ imposes the constraint

$$
2 N_{2}-N_{1} \lesssim 31-4\left(N_{g}-3\right)
$$

A further limitation of the available parameters comes from the request that $M_{\mathrm{GUT}}$ is sufficiently smaller than the Planck mass, in order to trust field theory without quantum gravity, and sufficiently large to avoid quick proton decay. GUT gauge bosons with mass $M_{\mathrm{GUT}}$ mediate the decay $p \rightarrow \pi^{0} e^{+}$with lifetime

$$
\begin{aligned}
\tau\left(p \rightarrow \pi^{0} e^{+}\right) & =\frac{4 f_{\pi}^{2} M_{\mathrm{GUT}}^{4}}{\pi m_{p} \alpha_{\mathrm{GUT}}^{2}(1+D+F)^{2} \alpha_{N}^{2}\left[A_{R}^{2}+\left(1+\left|V_{u d}\right|^{2}\right)^{2} A_{L}^{2}\right]} \\
& =\left(\frac{M_{\mathrm{GUT}}}{10^{16} \mathrm{GeV}}\right)^{4}\left(\frac{1 / 35}{\alpha_{\mathrm{GUT}}}\right)^{2}\left(\frac{0.015 \mathrm{GeV}^{3}}{\alpha_{N}}\right)^{2}\left(\frac{5}{A_{L}}\right)^{2} 4.4 \times 10^{34} \mathrm{yr} .
\end{aligned}
$$

each $S U(3)$ factor. By using eq. 7 we then find

$$
\begin{gathered}
N_{3}=q_{3} D_{2}, \quad N_{2}=q_{2} D_{3}, \\
\frac{5}{2} N_{1}=\frac{3}{4} \operatorname{Tr} Y^{2}=\left(3 p_{3}+q_{3}\right) D_{2}+\left(2 p_{2}+q_{2}\right) D_{3}-2 r_{2} r_{3},
\end{gathered}
$$

where $p_{2}, q_{2}, r_{2}, p_{3}, q_{3}, r_{3}$ are given by eqs. [7. Therefore, $N_{3}, N_{2}$ and $5 N_{1} / 2$ are integers. 
Here $f_{\pi}=131 \mathrm{MeV}$, the chiral Lagrangian factor is $(1+D+F)=2.25$, and we have taken the operator renormalization factors $A_{L}=A_{R}=5$. For the hadronic matix element $\alpha_{N}$, we take the lattice result [5] $\alpha_{N}=0.015 \mathrm{GeV}^{3}$. From the Super-Kamiokande limit [6] $\tau\left(p \rightarrow \pi^{0} e^{+}\right)>5.3 \times 10^{33} \mathrm{yr}$, we obtain

$$
M_{\mathrm{GUT}}>\left(\frac{\alpha_{\mathrm{GUT}}}{1 / 35}\right)^{1 / 2}\left(\frac{\alpha_{N}}{0.015 \mathrm{GeV}^{3}}\right)^{1 / 2}\left(\frac{A_{L}}{5}\right)^{1 / 2} 6 \times 10^{15} \mathrm{GeV}
$$

The measured value of $\alpha_{s}\left(M_{Z}\right)$ and the upper and lower bounds on $M_{\mathrm{GUT}}$ (given by the vertical lines in fig. (1) select an allowed region in the space of the Dynkin indices of new matter. From this result, we can already conclude that one-step unification cannot be achieved by adding to the SM only particles carrying no colour charges. Indeed, for $N_{3}=0$ we find an upper bound on $M_{\mathrm{GUT}}$, which is maximized for $N_{1}=0$, corresponding to $M_{\mathrm{GUT}}<2 \times 10^{14} \mathrm{GeV}$. This bound becomes much more stringent if the new weak particles carry hypercharge. At any rate, the upper bound on $M_{\mathrm{GUT}}$ is inconsistent with eq. (12). Also from fig. 1 it is apparent that there is no solution when $N_{2}=0$. Therefore unification requires a new set of particles carrying both colour and weak charges.

Since the variables $N_{i}$ can only take discrete values, only certain points in the region of fig. 1] are physical. To procede, we elaborate on the new particle content. We start by considering only fermions in real irreps, as this appears to be the most interesting physical case. Fermions in chiral representations, from incomplete GUT multiplets, generically introduce anomalies. Moreover, they can only get mass from electroweak symmetry breaking, and they are typically ruled out either by direct experimental searches or by their virtual effects on precision measurements. New light scalars, besides the Higgs, imply further fine-tunings and therefore, for simplicity, it is preferable to exclude them. At any rate, we will later generalize our results to the case of chiral fermions and scalars.

Since eventually we want to unify the theory, the new particles should belong to some (incomplete) GUT multiplet. This restricts the allowed quantum numbers. If, for simplicity, we consider the fermions to be part of the $5+\overline{5}, 10+\overline{10}, 15+\overline{15}, 24$ irreps of $S U(5)$ and exclude higher dimensional irreps, the possible new particles are

$$
\begin{aligned}
Q=(3,2,1 / 3)+(\overline{3}, 2,-1 / 3) & U=(3,1,4 / 3)+(\overline{3}, 1,-4 / 3) \\
D=(3,1,-2 / 3)+(\overline{3}, 1,2 / 3) & L=(1,2,1)+(1,2,-1) \\
E=(1,1,2)+(1,1,-2) & V=(1,3,0) \\
G=(8,1,0) & X=(3,2,-5 / 3)+(\overline{3}, 2,5 / 3) \\
T=(1,3,2)+(1,3,-2) & S=(6,1,-4 / 3)+(\overline{6}, 1,4 / 3) .
\end{aligned}
$$


Introducing a generic number $n_{a}$ of multiplets belonging to the irreps $a$ listed above, we find that their contributions to the $\beta$-function are

$$
\begin{aligned}
& N_{2}-N_{3}=2 A \\
& \frac{5}{2}\left(N_{2}-N_{1}\right)=2(A+3 B) \\
& N_{2}=2\left(3 n_{Q}+n_{L}+2 n_{V}+3 n_{X}+4 n_{T}\right) \\
& A \equiv n_{Q}-n_{U}-n_{D}+n_{L}+2 n_{V}-3 n_{G}+n_{X}+4 n_{T}-5 n_{S} \\
& B \equiv 2 n_{Q}-n_{U}-n_{E}+n_{V}+n_{G}-2 n_{X}-n_{T}-n_{S}
\end{aligned}
$$

Therefore, the allowed points of the plane in fig. 1 1 are those obtained from eqs. (14) and (15) for integers (positive or negative) values of $A$ and $B$. This is actually true independently of the fermion content of the theory, provided that the fermion representation is real and the new particles belong to some (incomplete) $S U(5)$ multiplet (see footnote 2).

Negative values of $A$ correspond to solutions where $M_{\mathrm{GUT}}>M_{\mathrm{Pl}}$, which we discard. For $A=1$, we find a solution with $B=1$, corresponding to $\alpha_{s}\left(M_{Z}\right)=0.120$ and $M_{\mathrm{GUT}}=$ $9.8 \times 10^{14}$. The minimal particle content is given by $(E+Q)$ or $(D+V)$. The case $(D+V)$ is particularly interesting, because $V$ contains a dark matter candidate, linking the new particles to the $\mathrm{TeV}$ scale. However, even though $\alpha_{s}\left(M_{Z}\right)$ is in agreement with measurements, $M_{\mathrm{GUT}}$ is in conflict with the proton-decay bound in eq. (12). We will discard this solution.

We are left only with the case $A=0$. The solution for $B=2$ gives $\alpha_{s}\left(M_{Z}\right)=0.102$ and $M_{\mathrm{GUT}}=1.6 \times 10^{16}$. The value of $M_{\mathrm{GUT}}$ is consistent with eq. (12), but $\alpha_{s}\left(M_{Z}\right)$ is rather low and a certain amount of threshold effects are beneficial, as we will discuss in the next section. Possible particle contents can be obtained by solving eqs.(17) and (18). The minimal options are $(Q+D),(L+V+G),(V+2 G+T),(U+2 V+G),(2 Q+2 U)$, or $(Q+2 D+L)$. Those containing $L$ or $V$ have a dark matter candidate. The case $(Q+D)$ has been proposed in ref. [7] as a cure to the discrepancy between measurements and SM prediction of the b-quark forward-backward asymmetry. The particle content of $(L+V+G)$ corresponds to the supersymmetric SM with a single Higgs doublet: it is thus the case advocated in ref. [3]. Indeed, we can identify $L$ with the (Dirac) higgsino components, interpret $V$ and $G$ as the $\mathrm{W}$-ino and gluino, and add a gauge singlet B-ino (which does not affect the gauge-coupling evolution at one-loop) to complete the gaugino spectrum.

The generalization of these results is now straightforward. Chiral fermions can contribute to $A$ and $B$ with half-integers and complex scalars with multiples of $1 / 4$. The solutions with acceptable $M_{\mathrm{GUT}}$ and $\alpha_{s}\left(M_{Z}\right)$ (within 2- $\sigma$ ) are collected in table 1 . The explicit particle 


\begin{tabular}{|c|c|c|c|c|c|}
\hline \hline$A$ & $B$ & $N_{2}-N_{3}$ & $\frac{5}{2}\left(N_{2}-N_{1}\right)$ & $\alpha_{s}\left(M_{Z}\right)$ & $M_{\mathrm{GUT}}[\mathrm{GeV}]$ \\
\hline$-\frac{1}{2}$ & $\frac{13}{4}$ & -1 & 18.5 & 0.121 & $6.2 \times 10^{18}$ \\
$-\frac{1}{4}$ & $\frac{11}{4}$ & -0.5 & 16 & 0.115 & $5.0 \times 10^{17}$ \\
0 & $\frac{5}{2}$ & 0 & 15 & 0.120 & $2.0 \times 10^{17}$ \\
$\frac{1}{4}$ & 2 & 0.5 & 12.5 & 0.115 & $2.4 \times 10^{16}$ \\
$\frac{1}{2}$ & $\frac{7}{4}$ & 1 & 11.5 & 0.120 & $1.1 \times 10^{16}$ \\
$\frac{3}{4}$ & $\frac{5}{4}$ & 1.5 & 9 & 0.115 & $1.9 \times 10^{15}$ \\
\hline \hline
\end{tabular}

Table 1: $N_{1,2,3}$ are the Dynkin indices of the particle content of new matter (including scalars) necessary to achieve gauge-coupling unification in the SM. The corresponding predictions for $\alpha_{s}\left(M_{Z}\right)$ and $M_{\mathrm{GUT}}$ are also listed. The values of $A$ and $B$ and eqs. (17) and (18) determine the particle multiplets of new matter.

content of the solution listed in table 1 can be obtained from eqs. (17) and (18). Notice that the case $\left(N_{2}-N_{3}\right)=1 / 2$ and $\left(N_{2}-N_{1}\right) 5 / 2=25 / 2$ corresponds to the usual supersymmetric extension of the SM with two Higgs doublets. From the results in table 1 we observe that some of the solutions have a value of $M_{\mathrm{GUT}}$ close to the experimental limit and therefore predict a measurable proton-decay rate. Two-loop renormalization group and threshold effects can give shifts of $\alpha_{s}\left(M_{Z}\right)$ even larger than the experimental error. These effects split the degeneracy of the various models, since they depend on the matter content through more parameters than simply $\left(N_{2}-N_{3}\right)$ and $\left(N_{2}-N_{1}\right)$. For instance, in the minimal supersymmetric model, two-loop running effects amount to a shift $\Delta \alpha_{s}\left(M_{Z}\right)=+0.011$. Low-energy threshold corrections depend on the detailed mass spectrum. The contributions from individual particles can be very significant, although in the minimal supersymmetric model a certain amount of cancellation among the various contributions typically occurs.

In summary, we have classified the particle content of new matter at the electroweak scale, necessary to achieve gauge-coupling unification in the SM (see also ref. [8]). We have found several class of solutions. However, if we require the presence of only fermions in real irreps, there is only one class of solutions. Although there is a large degeneracy of models with the same values of $\left(N_{2}-N_{3}\right)$ and $\left(N_{2}-N_{1}\right)$, therefore belonging to the same class, the case of Split Supersymmetry proposed in ref. [3] is the case of minimal field content with a dark matter candidate.

\subsection{Split Supersymmetry versus Non-Supersymmetric Solutions}

It is likely that supersymmetry plays a rôle in a consistent theory of gravity, like superstrings. But, if we abandon the naturalness problem, the rationale for low-energy supersymmetry is lost, since the preferred superstring vacuum may well completely break supersymmetry. 
Nevertheless, we want to argue that the case of Split Supersymmetry looks particularly intersting, when compared with the other non-supersymmetric solutions to gauge unification that we have found in the previous section.

In general, once we introduce new particles at the weak scale to obtain gauge-coupling unification, we encounter several problems. Split Supersymmetry seems to have partial answers to several of them. Let us briefly analyse them.

(i) Splitting of GUT irreps. Gauge-coupling unification requires the addition of new incomplete GUT multiplets. It is not always natural in a GUT to obtain the appropriate content of light particles. This is nothing else than the old doublet-triplet splitting problem for the Higgs multiplet. In Split Supersymmetry, once the necessary Higgs doublet-triplet splitting is achieved, no other incomplete matter multiplets are necessary. Supersymmetry plays a crucial rôle in relating the content of gauginos to the incomplete irrep of gauge bosons, and higgsinos to Higgs.

(ii) Light fermions. To maintain vector-like fermions light, the theory should posess some approximate global symmetries. Supersymmetry has the advantage of having some of this symmetries already built in. Gaugino and higgsino masses can be protected, even after soft supersymmetry breaking, by an $R$-symmetry and a PQ symmetry, related to the origin of the $\mu$ term [9]. We will show that actually, in the effective theory, only a linear combination of the two symmetries survives, but this is sufficient to protect gaugino and higgsino masses.

(iii) Existence and stability of dark matter. In this framework, dark matter provides the link with the electroweak scale. Therefore the existence and stability of a weakly-interacting neutral particle is a crucial ingredient. Supersymmetry has these features built in, because of the $R$-parity, which can be a low-energy consequence of a GUT gauge symmetry.

(iv) Instability of coloured particles. We have seen that the condition for one-step unification and proton stability requires the existence of new coloured particles at the electroweak scale. If these particles are stable, they could be present today, bound in nuclei, and they would appear as anomalously heavy isotopes. Their relic abundance is quite uncertain, because it depends on the mechanism of hadronization and of nuclear binding [10]. However, even allowing for the most conservative estimates, stable coloured particles are ruled out by searches for heavy hydrogen isotopes, which excludes that their number per nucleon is larger than $10^{-28}$ for a mass up to $1 \mathrm{TeV}$ [1] and $10^{-20}$ up to $10 \mathrm{TeV}[12$. The decay of the new coloured particles could arise from mixing with ordinary quarks through renormalizable interactions. However this mixing typically introduces unwanted flavour violations, and it is tightly constrained. If the decay occurs through a non-renormalizable 4-fermion interaction 
with scale $\Lambda$, the lifetime of the coloured particle with mass $M$ is

$$
\tau \simeq(4 \pi)^{3} \frac{\Lambda^{4}}{M^{5}} \simeq\left(\frac{\mathrm{TeV}}{M}\right)^{5}\left(\frac{\Lambda}{10^{13} \mathrm{GeV}}\right)^{4} 0.4 \mathrm{Gyr} .
$$

Thus, the decay of the coloured particle requires either a small parameter or a new threshold at an intermediate scale smaller than about $10^{13} \mathrm{GeV}$, which can modify the gauge-coupling evolution. In Split Supersymmetry, the intermediate threshold is provided by the squark and slepton masses, which mediate gluino decay. This threshold consists of a complete GUT irrep (apart from the heavy Higgs doublet) and therefore does not (much) affect the unification condition. As long as the mechanism of supersymmetry breaking explains the existence of two widely separated scales, the gluino stability does not pose a problem.

Because of the interesting features of Split Supersymmetry, we proceed to investigate it in more detail.

\section{The Structure of Split Supersymmetry}

The spectrum of Split Supersymmetry contains the higgsino components $\tilde{H}_{u, d}$, the gluino $(\tilde{g})$, the $\mathrm{W}$-ino $(\tilde{W})$ and B-ino $(\tilde{B})$, and the SM particles with one Higgs doublet. The most general renormalizable Lagrangian with a matter parity, besides gauge-invariant kinetic terms, is given by

$$
\begin{aligned}
\mathcal{L}= & m^{2} H^{\dagger} H-\frac{\lambda}{2}\left(H^{\dagger} H\right)^{2}-\left[h_{i j}^{u} \bar{q}_{j} u_{i} \epsilon H^{*}+h_{i j}^{d} \bar{q}_{j} d_{i} H+h_{i j}^{e} \bar{\ell}_{j} e_{i} H\right. \\
& +\frac{M_{3}}{2} \tilde{g}^{A} \tilde{g}^{A}+\frac{M_{2}}{2} \tilde{W}^{a} \tilde{W}^{a}+\frac{M_{1}}{2} \tilde{B} \tilde{B}+\mu \tilde{H}_{u}^{T} \epsilon \tilde{H}_{d} \\
& \left.+\frac{H^{\dagger}}{\sqrt{2}}\left(\tilde{g}_{u} \sigma^{a} \tilde{W}^{a}+\tilde{g}_{u}^{\prime} \tilde{B}\right) \tilde{H}_{u}+\frac{H^{T} \epsilon}{\sqrt{2}}\left(-\tilde{g}_{d} \sigma^{a} \tilde{W}^{a}+\tilde{g}_{d}^{\prime} \tilde{B}\right) \tilde{H}_{d}+\text { h.c. }\right],
\end{aligned}
$$

where $\epsilon=i \sigma_{2}$.

The Lagrangian in eq. (20) describes the effective theory obtained by removing squarks, sleptons, charged and pseudoscalar Higgs from the supersymmetric SM. These states are assumed to be heavy and, for simplicity, we will assume them to be all degenerate with mass $\tilde{m}$. The coupling constants of the effective theory at the scale $\tilde{m}$ are obtained by matching the Lagrangian in eq. (20) with the interaction terms of the supersymmetric Higgs doublets $H_{u}$ and $H_{d}$,

$$
\begin{aligned}
\mathcal{L}_{\text {susy }}= & -\frac{g^{2}}{8}\left(H_{u}^{\dagger} \sigma^{a} H_{u}+H_{d}^{\dagger} \sigma^{a} H_{d}\right)^{2}-\frac{g^{\prime 2}}{8}\left(H_{u}^{\dagger} H_{u}-H_{d}^{\dagger} H_{d}\right)^{2} \\
& +\lambda_{i j}^{u} H_{u}^{T} \epsilon \bar{u}_{i} q_{j}-\lambda_{i j}^{d} H_{d}^{T} \epsilon \bar{d}_{i} q_{j}-\lambda_{i j}^{e} H_{e}^{T} \epsilon \bar{e}_{i} \ell_{j}
\end{aligned}
$$




$$
-\frac{H_{u}^{\dagger}}{\sqrt{2}}\left(g \sigma^{a} \tilde{W}^{a}+g^{\prime} \tilde{B}\right) \tilde{H}_{u}-\frac{H_{d}^{\dagger}}{\sqrt{2}}\left(g \sigma^{a} \tilde{W}^{a}-g^{\prime} \tilde{B}\right) \tilde{H}_{d}+\text { h.c. }
$$

Once the Higgs doublet $H=-\cos \beta \epsilon H_{d}^{*}+\sin \beta H_{u}$ is fine-tuned to have small mass term, the matching conditions of the coupling constants in eq. (20) at the scale $\tilde{m}$ are obtained by replacing $H_{u} \rightarrow \sin \beta H, H_{d} \rightarrow \cos \beta \epsilon H^{*}$ in eq. (21):

$$
\begin{aligned}
\lambda(\tilde{m})= & \frac{\left[g^{2}(\tilde{m})+g^{\prime 2}(\tilde{m})\right]}{4} \cos ^{2} 2 \beta, \\
h_{i j}^{u}(\tilde{m})=\lambda_{i j}^{u *}(\tilde{m}) \sin \beta, & h_{i j}^{d, e}(\tilde{m})=\lambda_{i j}^{d, e *}(\tilde{m}) \cos \beta, \\
\tilde{g}_{u}(\tilde{m})=g(\tilde{m}) \sin \beta, & \tilde{g}_{d}(\tilde{m})=g(\tilde{m}) \cos \beta, \\
\tilde{g}_{u}^{\prime}(\tilde{m})=g^{\prime}(\tilde{m}) \sin \beta, \quad & \tilde{g}_{d}^{\prime}(\tilde{m})=g^{\prime}(\tilde{m}) \cos \beta .
\end{aligned}
$$

In the context of a supersymmetric theory it is possible to argue that the gaugino masses $M_{i}$ or the higgsino mass $\mu$ are much smaller than the typical scale because they are protected by an $R$-symmetry and a PQ symmetry, respectively. However, the symmetries of the effective Lagrangian in eq. (20) are not enhanced if we set $M_{i}=0$ or $\mu=0$, separately. This is because supersymmetry has been explicitly broken by eliminating the scalar fields. Nevertheless, if we simultaneously set $M_{i}=\mu=0$, the effective theory remains invariant under the product of an $R$-symmetry (with $R$-charges $R\left[H_{u}\right]=2, R\left[H_{d}\right]=0$ ) and a supersymmetric PQ symmetry (with charges $P Q\left[H_{u}\right]=P Q\left[H_{d}\right]=-1$ ). In other words, eq. (20) is invariant under a global $U(1)$ symmetry with charges $S[\tilde{B}]=S[\tilde{W}]=S[H]=-S\left[\tilde{H}_{d}\right] / 2$, $S\left[\tilde{H}_{u}\right]=0$, and with quarks and leptons with the appropriate charges. This symmetry is spontaneously broken by the Higgs vev and explicitly broken by $M_{i}$ or $\mu$.

This result shows that the choice of keeping only light gauginos or light higgsinos, but not both of them simultaneously, is not radiatively stable. It is interesting that the only consistent choice (forgetting the tuning of the Higgs mass) of splitting the supersymmetric spectrum is the one that successfully reproduces gauge-coupling unification. Another consequence of this result is that the $\mu$ parameter mixes with $M_{1,2}$ under renormalization effects, as discussed in sect. 3.2

A particular situation occurs when $\tan \beta \rightarrow \infty$, since $h^{d}, h^{e}, \tilde{g}_{d}, \tilde{g}_{d}^{\prime}$ vanish in this limit. Then eq. (201), for $\mu=0$, has an additional global symmetry (with charges $([\tilde{B}]=[\tilde{W}]=0$, $\left.[H]=\left[\tilde{H}_{u}\right]=\left[\tilde{H}_{d}\right]\right)$. When we set $M_{i}=0$ and keep $\mu$ non-vanishing, we find a different global symmetry (with charges $\left([\tilde{B}]=[\tilde{W}]=[H] / 2=\left[\tilde{H}_{u}\right]=-\left[\tilde{H}_{d}\right]\right)$. Therefore, we expect no renormalization mixing between $M_{1,2}$ and $\mu$ in the limit of large $\tan \beta$. 


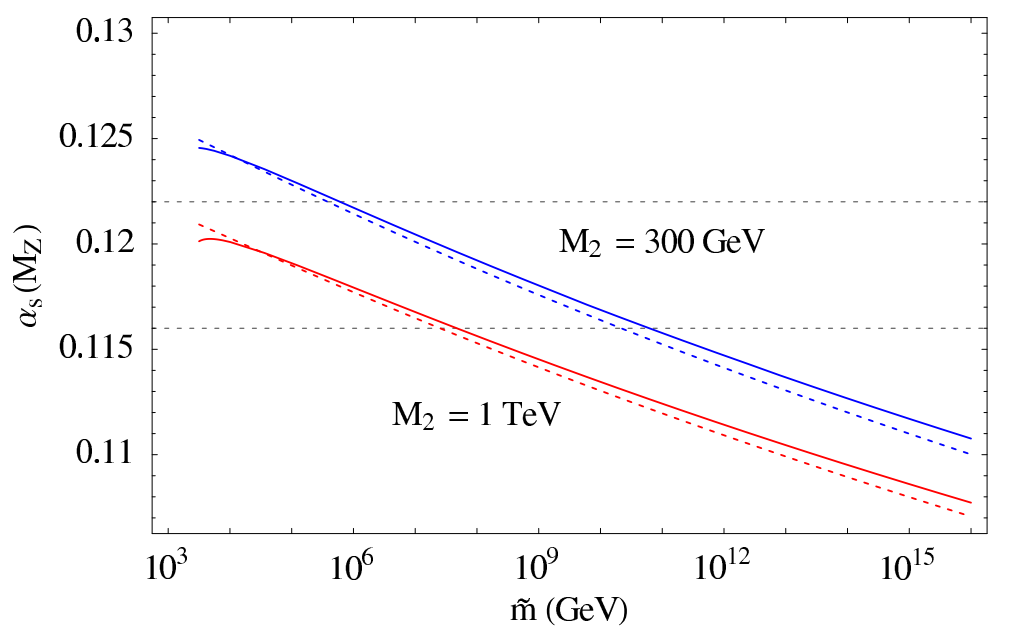

Figure 2: The unification prediction for $\alpha_{s}\left(M_{Z}\right)$ as a function of $\tilde{m}$. The solid line corresponds to $\tan \beta=50$ and the dashed line to $\tan \beta=1.5$. The gaugino and higgsino thresholds are taken by assuming gaugino mass unification, and $\mu=M_{2}$. The results for $M_{2}=300 \mathrm{GeV}$ and $1 \mathrm{TeV}$ are shown.

\subsection{Unification}

To make a precise assessment on gauge-coupling constant unification in Split Supersymmetry, it is necessary to study the 2-loop renormalization group evolution, including one-loop threshold effects. Between the unified scale $M_{\mathrm{GUT}}$ and the scale of heavy scalars $\tilde{m}$, the theory is exactly supersymmetric. Below the scale $\tilde{m}$, we use the spectrum of Split Supersymmetry, with gauginos and higgsinos included in the 2-loop evolution. Then we include separate threholds for the gluino $M_{3}$ and for weak gaugino and higgsino masses, and properly evolve between the two scales. Thresholds of the top quark and SM gauge bosons are taken into account in the usual way [13. The relevant renormalization-group equation are given in the appendix. We use the same SM input values used in sect. 2.

The prediction of $\alpha_{s}\left(M_{Z}\right)$, as a function of the intermediate scale $\tilde{m}$, is shown in fig. 2 , We have shown our results for values of $\tilde{m}$ as large as $M_{\mathrm{GUT}}$, although the condition that the gluino lifetime is shorter than the age of the universe requires $\tilde{m} \lesssim 10^{13} \mathrm{GeV}$. However, we recall that the limit from the search for heavy isotopes is valid only up to a gluino mass of $10 \mathrm{TeV}$.

A value of $\tilde{m}$ larger than in conventional supersymmetric models improves the agreement between the theoretical prediction and experimental data. However, because of the theoretical uncertainty due to unknown GUT thresholds and supersymmetric thresholds at the scale $\tilde{m}$, it is not possible to extract firm bounds on the parameters. A dependence of $\alpha_{s}\left(M_{Z}\right)$ 

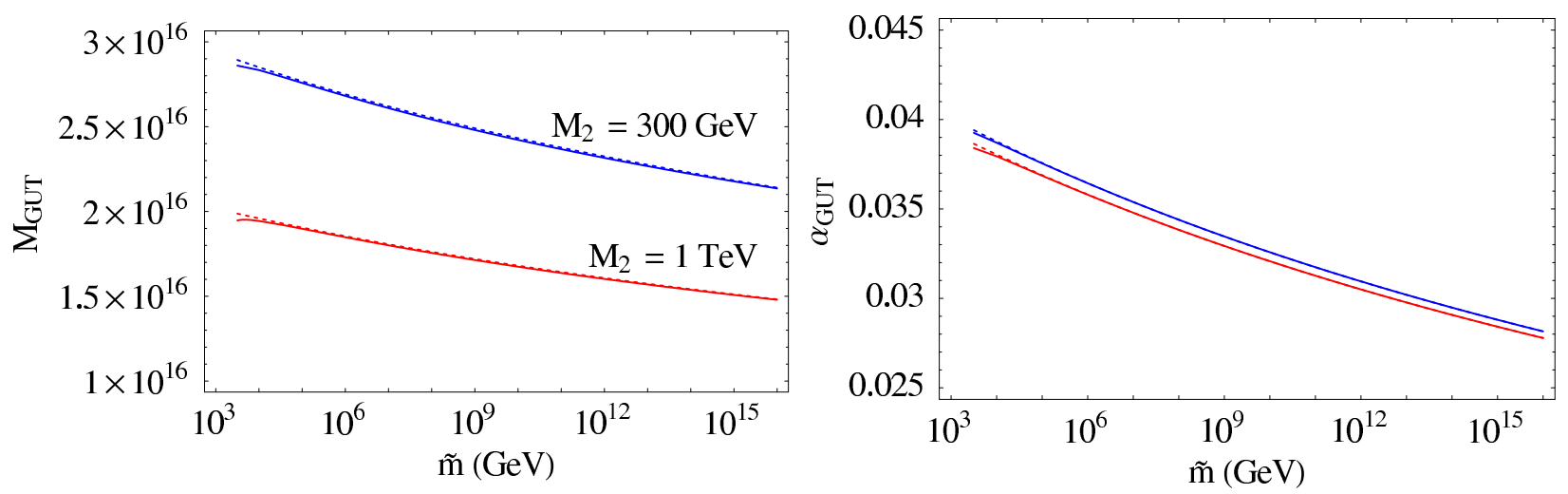

Figure 3: The unification prediction for $M_{\mathrm{GUT}}$ and $\alpha_{\mathrm{GUT}}$ as functions of $\tilde{m}$. The solid line corresponds to $\tan \beta=50$ and the dashed line to $\tan \beta=1.5$. The gaugino and higgsino thresholds are taken by assuming gaugino mass unification, and $\mu=M_{2}$. The results for $M_{2}=300 \mathrm{GeV}$ and $1 \mathrm{TeV}$ are shown.

on $\tan \beta$ arises from two-loop effects proportional to the Yukawa couplings and the gaugino couplings $\tilde{g}$, but the numerical contribution is marginal. On the other hand, the effect of the gaugino and higgsino threshold is important.

The prediction for $M_{\mathrm{GUT}}$ is shown in fig. 3. The unified mass decreases as $\tilde{m}$ grows, but the proton-decay rate from dimension-6 operators remains unobservably small, at present. We recall that proton decay through dimension-5 operators is suppressed by the large squark mass. The value of $\alpha_{\mathrm{GUT}}$ decreases with $\tilde{m}$, because of the smaller particle content of Split Supersymmetry with respect to the ordinary case, as shown in fig. 3 .

Before discussing the unification of bottom and $\tau$ masses, let us discuss the running of the top Yukawa coupling. It is well known that in the MSSM the Landau pole sets an upper limit on the top coupling, which translates in a lower limit on $\tan \beta$. In split supersymmetry, the Landau pole is met before the unification scale for lower values of $\tan \beta$. This happens for two reasons. First, below $\tilde{m}$, the Yukawa coupling belongs to a theory with a single Higgs doublet and it is smaller by a factor $\sin \beta$ than in the two-Higgs theory. Second, the contribution of the top coupling to its evolution is smaller (see equations in the appendix). As a consequence, the value of the coupling at the matching with the supersymmetric theory is smaller, and this allows a smaller value of $\tan \beta$. Such low values of $\tan \beta$ are not necessarily ruled out in this scenario, as the Higgs mass can be made sufficiently large even for low values of $\tan \beta$ (see sect. 3.4).

Since the model has a successful gauge-coupling unification, it is natural to study if also the bottom and $\tau$ masses can unify at the same scale. We take the bottom-quark $\overline{\mathrm{MS}}$ running 

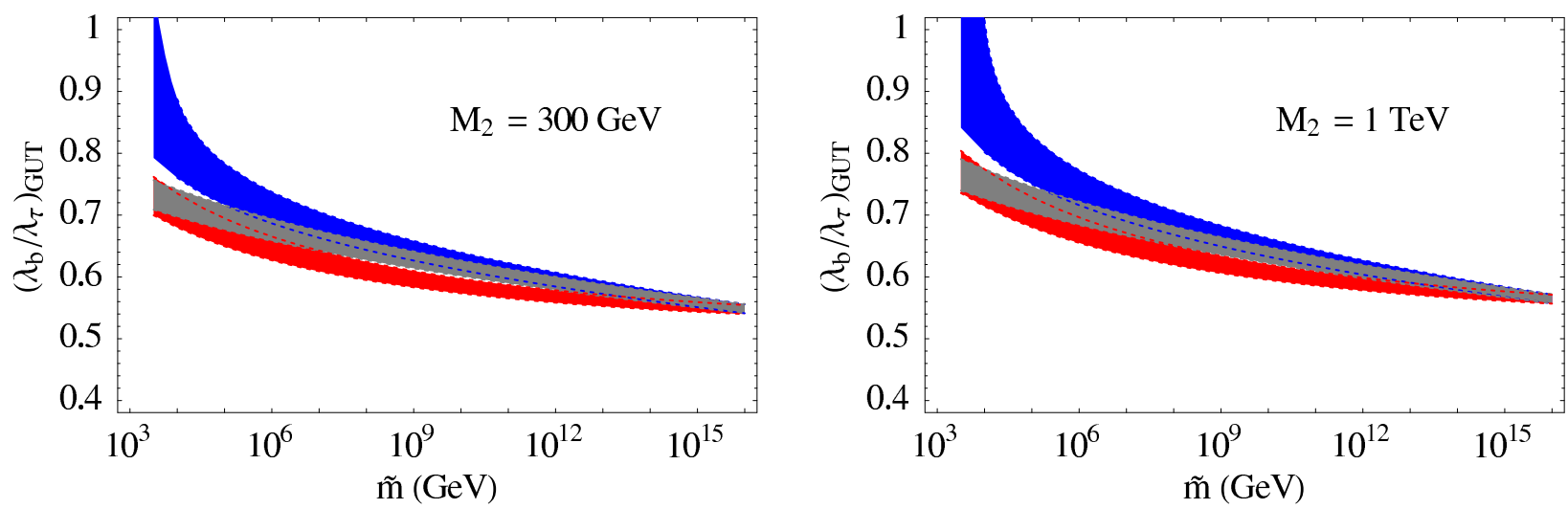

Figure 4: The Yukawa-coupling ratio $\lambda_{b} / \lambda_{\tau}$ evaluated at $M_{\mathrm{GUT}}$. The gaugino and higgsino thresholds are taken by assuming gaugino mass unification, and $\mu=M_{2}$. The results for $M_{2}=300 \mathrm{GeV}$ and $1 \mathrm{TeV}$ are shown. The bands correspond to $1-\sigma$ uncertainties in $m_{b}$ and $m_{t}$, and $\tan \beta=1.5,10,50$, from top to bottom.

mass $m_{b}\left(m_{b}\right)=4.2 \pm 0.1 \mathrm{GeV}$. Although the effective theory has only one Higgs doublet, the ratio $m_{b} / m_{\tau}$ depends on $\tan \beta$ because of the running above $\tilde{m}$. In fig. 4 we show the Yukawa-coupling ratio $\lambda_{b} / \lambda_{\tau}$ at the unification scale, as a function of $\tilde{m}$, for different values of $\tan \beta$. Here the trend with $\tilde{m}$ is the opposite than for the prediction of $\alpha_{s}\left(M_{Z}\right)$, and heavy scalars make $b-\tau$ unification more difficult for a given $\tan \beta$. For large $\tan \beta$ this is also because in Split Supersymmetry one can not rely on sizable finite corrections at large $\tan \beta$ [14. Since the coefficient $A$ of the trilinear soft terms is forbidden by the same $R$ symmetry that protects gaugino masses, the large-tan $\beta$ corrections are suppressed by powers of the heavy-scalar masses. For small $\tan \beta$, one obtains lower values of $b-\tau$, as a consequence of the slower running of the top Yukawa coupling discussed above (the top Yukawa increases the value of the bottom mass and does not affect the tau mass).

Despite heavy scalars make $b-\tau$ unification more difficult at a given $\tan \beta$, the prospects for $b-\tau$ unification at low $\tan \beta$ in Split Supersymmetry are actually better than in the MSSM. In fact, in both cases the top Yukawa enhancement of $m_{b}$ can be made large enough by using values of $\tan \beta$ close enough to the Landau pole lower limit (as in the MSSM, this involves a degree of fine-tuning). In the MSSM, such values of $\tan \beta$ are excluded by the Higgs mass limit. Because of the slower top running, in Split Supersymmetry the values of $\tan \beta$ required to enhance $m_{b}$ are even lower. However, they are not excluded by the Higgs mass limits, as we will see later on. Therefore, $b-\tau$ unification at low $\tan \beta$ is not ruled out for heavy scalars, differently than in the MSSM. Note that we have neglected in our discussion the possibility of a contribution from neutrino Yukawa couplings [15] (which enhances the tau mass and therefore goes in the wrong direction). Also, the $m_{b} / m_{\tau}$ ratio at $M_{\mathrm{GUT}}$ can 

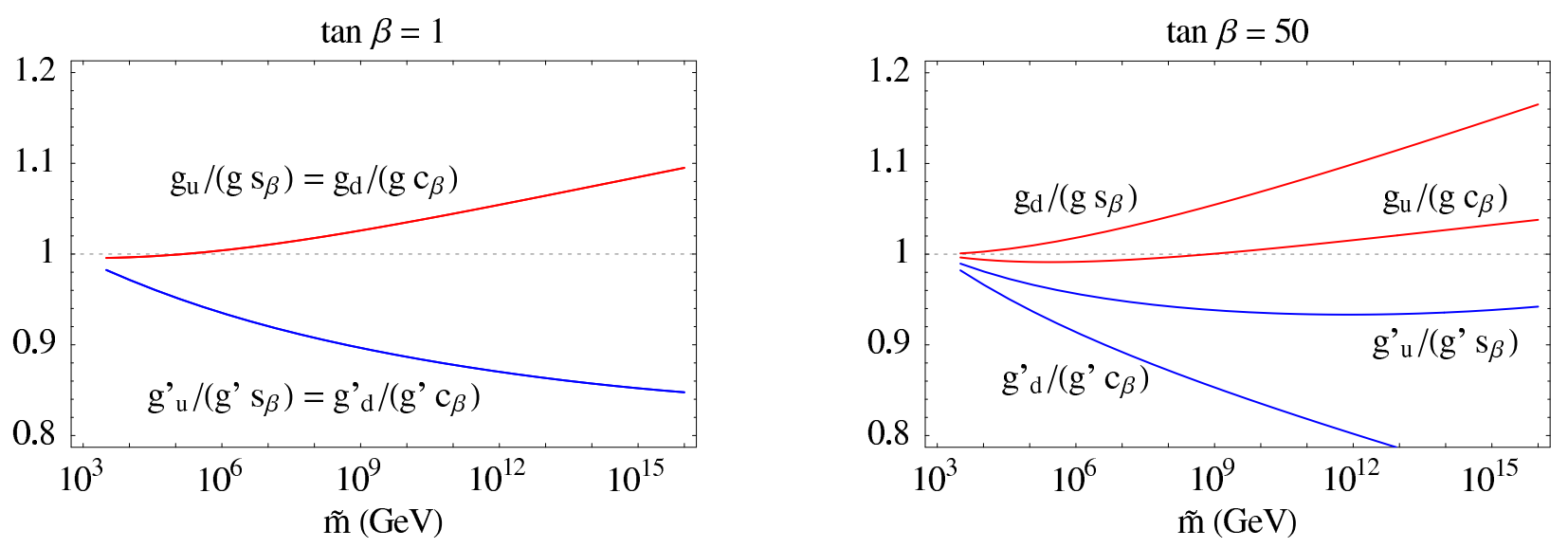

Figure 5: The gaugino couplings in units of the gauge couplings $\tilde{g}_{u} /(g \sin \beta), \tilde{g}_{d} /(g \cos \beta)$, $\tilde{g}_{u}^{\prime} /\left(g^{\prime} \sin \beta\right), \tilde{g}_{d}^{\prime} /\left(g^{\prime} \cos \beta\right)$ as functions of $\tilde{m}$, calculated at the weak scale. The left frame corresponds to $\tan \beta=1$ and the right one to $\tan \beta=50$.

be enhanced because of contribution from higher Higgs representations or because of the flavour structure of the Yukawa mass matrices.

\subsection{Gaugino Couplings}

A testable prediction of Split Supersymmetry is the deviation of the equality between gauge and gaugino couplings. This could be detected by precise measurements of the gaugino production cross section. Such tests have already been proposed in the case of low-energy supersymmetry [16]. The effect in Split Supersymmetry is much enhanced by the heaviness of squarks and slepton. A log resummation is required to compute the effect and the relevant renormalization-group equations are given in the appendix.

The gaugino couplings $\tilde{g}$, which satisfy the boundary conditions in eq. (25) at the scale $\tilde{m}$, are evolved to the weak scale. Numerical results are shown in fig. 5. To understand the behaviour, it is useful to take the analytic solution of the renormalization-group equations, in the limit of small $\ln (\tilde{m} / \bar{\mu})$, where $\bar{\mu}$ is the renormalization scale:

$$
\begin{aligned}
&\left.\frac{\tilde{g}_{u}}{g \sin \beta}\right|_{\bar{\mu}} \simeq 1+\frac{\ln (\tilde{m} / \bar{\mu})}{(4 \pi)^{2}}\left[\left(\frac{13}{3}+\frac{7}{4} \cos ^{2} \beta\right) g^{2}-\frac{3}{4} \cos ^{2} \beta g^{\prime 2}-3 h_{t}^{2}\right]_{\tilde{m}} \\
&\left.\frac{\tilde{g}_{u}^{\prime}}{g^{\prime} \sin \beta}\right|_{\bar{\mu}} \simeq 1+\frac{\ln (\tilde{m} / \bar{\mu})}{(4 \pi)^{2}}\left[-\frac{9}{4} \cos ^{2} \beta g^{2}+\left(7-\frac{3}{4} \cos ^{2} \beta\right) g^{\prime 2}-3 h_{t}^{2}\right]_{\tilde{m}} .
\end{aligned}
$$

The equations for $\tilde{g}_{d} /(g \cos \beta)$ and $\tilde{g}_{d}^{\prime} /\left(g^{\prime} \cos \beta\right)$ are obtained from eq. (26) and eq. (27), respectively, by replacing $\cos 2 \beta \rightarrow-\cos 2 \beta$ in the right-hand side. Gauge and top-quark 

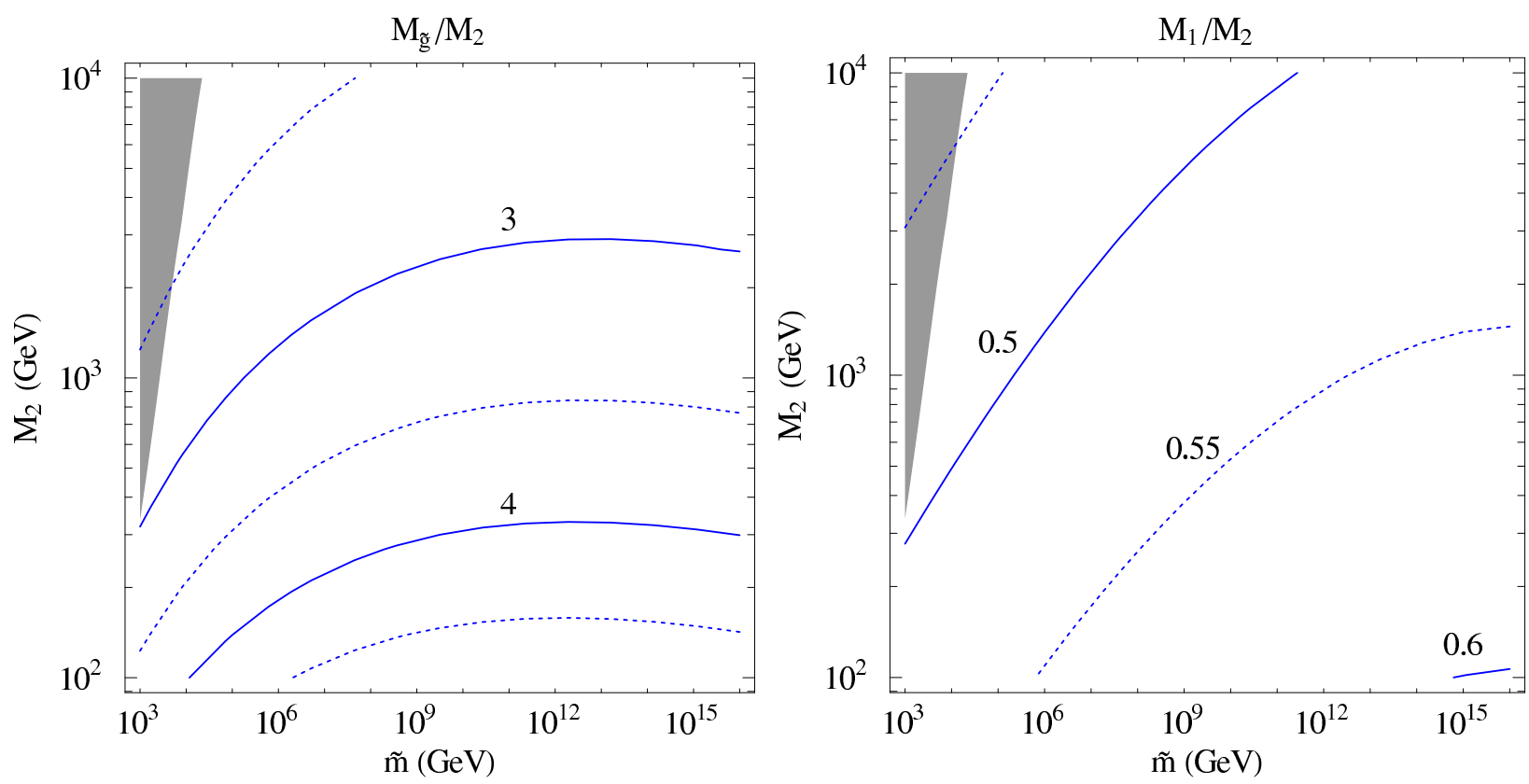

Figure 6: The ratios of the pole gluino mass $M_{\tilde{g}}$ to $M_{2}$ (left frame) and $M_{1} / M_{2}$ (right frame) as functions of $\tilde{m}$ and $M_{2}$, assuming gaugino mass unification. We have taken a large value of $\tan \beta$, so that the mixing between $M_{1,2}$ and $\mu$ is irrelevant. The shaded region corresponds to $\tilde{m}<M_{\tilde{g}}$.

Yukawa contribute to the running of the gaugino couplings with opposite signs. In $\tilde{g}_{u} /(g \sin \beta)$ and $\tilde{g}_{d} /(g \cos \beta)$, the $S U(2)$ contribution has a very large coefficient which overtakes the topYukawa effect. In $\tilde{g}_{u}^{\prime} /\left(g^{\prime} \sin \beta\right)$ and $\tilde{g}_{d}^{\prime} /\left(g^{\prime} \cos \beta\right)$, the most important gauge effects come from hypercharge, and top-quark effects tend to dominate. A significant cancellation of the two competing effects can occur in $\tilde{g}_{u}^{\prime} /\left(g^{\prime} \sin \beta\right)$ or $\tilde{g}_{u} /(g \sin \beta)$.

\subsection{Gaugino Masses}

We have computed the gluino pole mass with two-loop renormalization-group evolution and threshold effects, with the equations given in the appendix. To help the discussion, consider the one-loop expressions of the gaugino mass at the scale $\bar{\mu}$ (in the limit of large $\tan \beta$ )

$$
\begin{aligned}
& M_{3}(\bar{\mu})=M_{3}\left(M_{\mathrm{GUT}}\right) \frac{\alpha_{s}(\tilde{m})}{\alpha_{\mathrm{GUT}}}\left[\frac{\alpha_{s}(\bar{\mu})}{\alpha_{s}(\tilde{m})}\right]^{9 / 5}, \\
& M_{2}(\bar{\mu})=M_{2}\left(M_{\mathrm{GUT}}\right) \frac{g^{2}(\tilde{m})}{4 \pi \alpha_{\mathrm{GUT}}}\left[\frac{g^{2}(\bar{\mu})}{g^{2}(\tilde{m})}\right]^{33 / 7}, \\
& M_{1}(\bar{\mu})=M_{1}\left(M_{\mathrm{GUT}}\right) \frac{5 g^{\prime 2}(\tilde{m})}{12 \pi \alpha_{\mathrm{GUT}}}\left[\frac{g^{\prime 2}(\bar{\mu})}{g^{\prime 2}(\tilde{m})}\right]^{1 / 15} .
\end{aligned}
$$



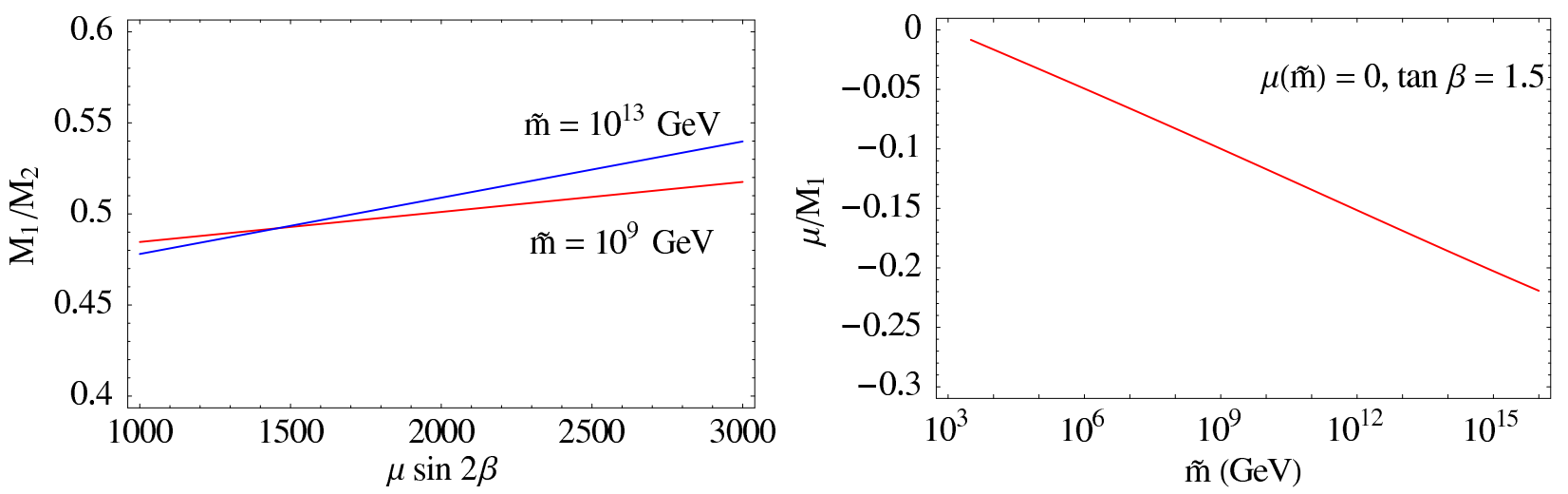

Figure 7: Left frame: the variation of the $M_{1} / M_{2}$ ratio with $\mu \sin 2 \beta$. Right frame: The value of the $\mu$ parameter at the weak scale in units of the smallest gaugino mass parameter $M_{1}$, assuming $\mu=0$ at the scale $\tilde{m}$, and gaugino mass unification. We have taken $\tan \beta=1.5$, since low values of $\tan \beta$ enhance the effect of radiative corrections.

The renormalization of the gaugino masses below $\tilde{m}$ is substantial. However, the ratio between gluino and weak-gaugino masses $M_{\tilde{g}} / M_{2}$ and the ratio $M_{2} / M_{1}$ do not significantly depend on $\tilde{m}$, as shown in fig. 6. This can be explained because the leading-logarithm evolution of the ratio $M_{i} / M_{j}$ is proportional to the difference between the numerical coefficients of the $M_{i}$ and $M_{j}$ anomalous dimensions. Therefore, intermediate thresholds of complete GUT multiplets (like squarks and sleptons at the scale $\tilde{m}$ ) have limited effect on the renormalization of the ratio $M_{i} / M_{j}$.

As explained above in terms of symmetries, we expect the unusual feature of mixing between $\mu$ and $M_{1,2}$ under renormalization effects. This is confirmed in the equations given in the appendix. For illustrative purposes, consider the approximate solutions for a renormalization scale $\mu$ not much smaller than $\tilde{m}$

$$
\begin{aligned}
& M_{2}(\bar{\mu}) \simeq \frac{\alpha M_{2}\left(M_{\mathrm{GUT}}\right)}{\sin ^{2} \theta_{W} \alpha_{\mathrm{GUT}}}\left[1+\frac{\ln (\tilde{m} / \bar{\mu})}{4 \pi}\left(\frac{11 \alpha}{\sin ^{2} \theta_{W}}-\frac{2 \alpha_{\mathrm{GUT}} \mu \sin 2 \beta}{M_{2}\left(M_{\mathrm{GUT}}\right)}\right)\right]_{\tilde{m}} \\
& M_{1}(\bar{\mu}) \simeq \frac{\alpha M_{1}\left(M_{\mathrm{GUT}}\right)}{\cos ^{2} \theta_{W} \alpha_{\mathrm{GUT}}}\left[\frac{5}{3}-\frac{\ln (\tilde{m} / \bar{\mu})}{4 \pi}\left(\frac{5 \alpha}{3 \cos ^{2} \theta_{W}}+\frac{2 \alpha_{\mathrm{GUT}} \mu \sin 2 \beta}{M_{1}\left(M_{\mathrm{GUT}}\right)}\right)\right]_{\tilde{m}},
\end{aligned}
$$

where couplings on the right-hand side are evaluated at the scale $\tilde{m}$. Notice that indeed, as explained above, the mixing disappears for large $\tan \beta$. Even for $\tan \beta \simeq 1$, the mixing effect is suppressed with respect to the renormalization of $M_{2}$. The numerical evaluation of the ratio $M_{1} / M_{2}$, using the equations given in the appendix, is shown in fig. 6. The effect of the mixing with $\mu$ is very limited, as exhibited in fig. [7.

Because of the mixing between $M_{1,2}$ and $\mu$, we could imagine to entirely generate $\mu$ by radiative effects. Therefore we consider the possibility that $\mu=0$ at the matching scale 

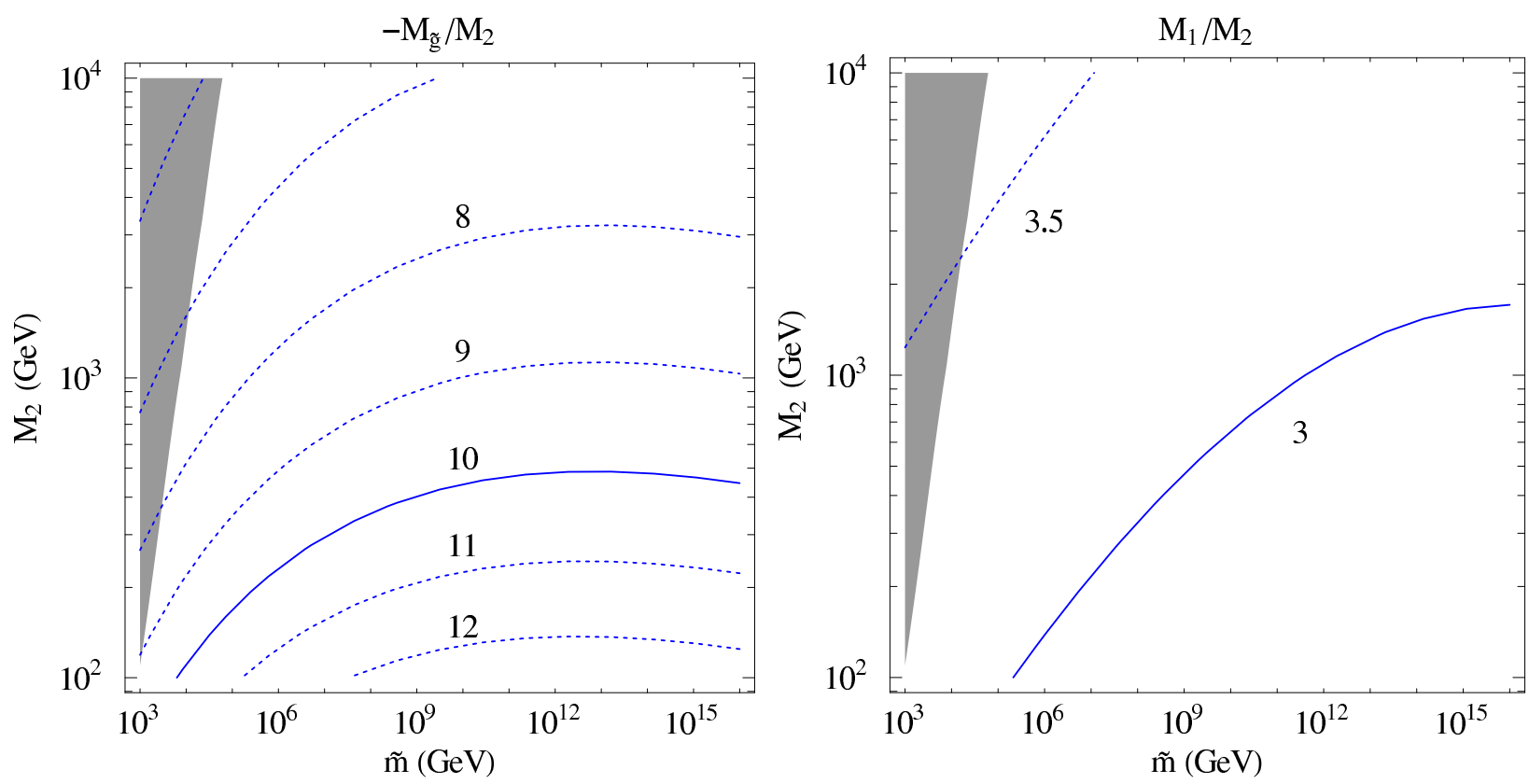

Figure 8: The ratios of the pole gluino mass $M_{\tilde{g}}$ to $M_{2}$ (left frame) and $M_{1} / M_{2}$ (right frame) as functions of $\tilde{m}$, assuming gaugino mass conditions from anomaly-mediation. We have taken a large value of $\tan \beta$, so that the mixing between $M_{1,2}$ and $\mu$ is irrelevant. The shaded region corresponds to $\tilde{m}<M_{\tilde{g}}$.

$\tilde{m}$, and we compute the low-energy value, with the results shown in fig. [7. We find that $\mu$ turns out to be significantly smaller than the gaugino masses, and therefore the lightest neutralino is a higgsino. In the next section, we will show that a dark-matter higgsino requires $\mu \simeq 1.0-1.2 \mathrm{TeV}$. Therefore, in this case, the whole spectrum is quite heavy.

So far we have considered a unification condition for gaugino masses. This appears quite justified, since unification is a crucial ingredient of this analysis. However, we can consider another interesting possibility. If supersymmetry breaking occurs in a sector without gauge singlets (as in models with dynamical symmetry breaking), than the leading contribution to gaugino masses comes from anomaly mediation [17]. The value of the gaugino masses traces the $\beta$-functions all the way down to the scale $\tilde{m}$, below which the spectrum is no longer supersymmetric. Therefore we consider the following gaugino-mass boundary conditions at the scale $\tilde{m}$

$$
M_{i}=\frac{\beta_{g_{i}}}{g_{i}} m_{3 / 2} .
$$

Here $\beta_{g_{i}}$ are the beta-functions of the gauge coupling $g_{i}$ (see appendix) and $m_{3 / 2}$ is the vev of the auxiliary component of the supergravity compensator field. The anomaly-mediation mass relations are modified by the non-supersymmetric running below the scale $\tilde{m}$. The 


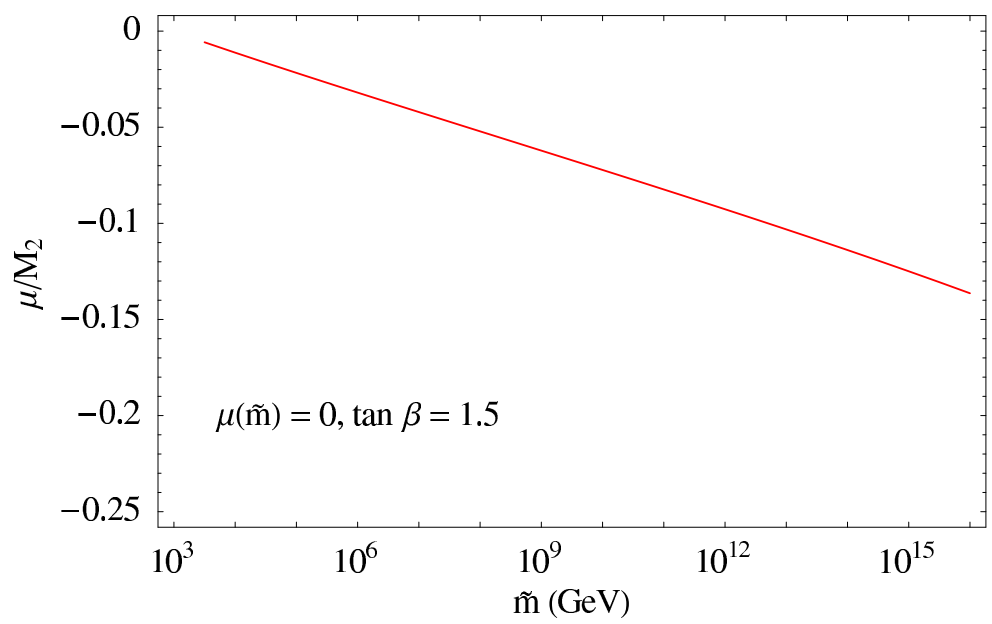

Figure 9: The value of the $\mu$ parameter at the weak scale in units of the smallest gaugino mass parameter $M_{2}$, assuming $\mu=0$ at the scale $\tilde{m}$, and anomaly-mediated relations for the gaugino masses. We have taken $\tan \beta=1.5$, which enhances the effect of radiative corrections.

ratio of the gaugino masses with anomaly-mediation boundary conditions are given in fig. 8, The $\mathrm{W}$-ino always remains the lightest gaugino.

We have also considered the case of a radiatively-generated $\mu$ parameter in Split $\mathrm{Su}$ persymmetry with anomaly mediation and the result in shown in fig. 9. As with gaugino unification, also in this case the higgsino turns out to be the lightest neutralino, but the hierarchy between $\mu$ and the gluino mass is amplified by the conditions of anomaly mediation.

\subsection{Higgs Mass}

The present lower bound on the SM Higgs mass, $m_{H}>114.4 \mathrm{GeV}$ at $95 \%$ CL [18, provides a strong constraint on the parameters of low-energy supersymmetry. This constraint is relaxed in Split Supersymmetry, because the Higgs boson mass receives large radiative correction in the evolution from $\tilde{m}$ to the weak scale. We have solved the relevant equations, contained in the appendix, and obtained the value of the Higgs mass shown in fig. 10, The band shows the uncertainty of the predictions due to the $1-\sigma$ error on the top-quark pole mass, $m_{t}=178.0 \pm$ $4.3 \mathrm{GeV}$. The Higgs-mass dependence on $\tan \beta$ comes primarily from the boundary condition in eq. (22), and to a lesser extent from the renormalization-group evolution. Because of the large renormalization effect, values of $\tan \beta$ equal to 1 are allowed, but they require large values of $\tilde{m}$ and low values of gaugino and higgsino masses. 


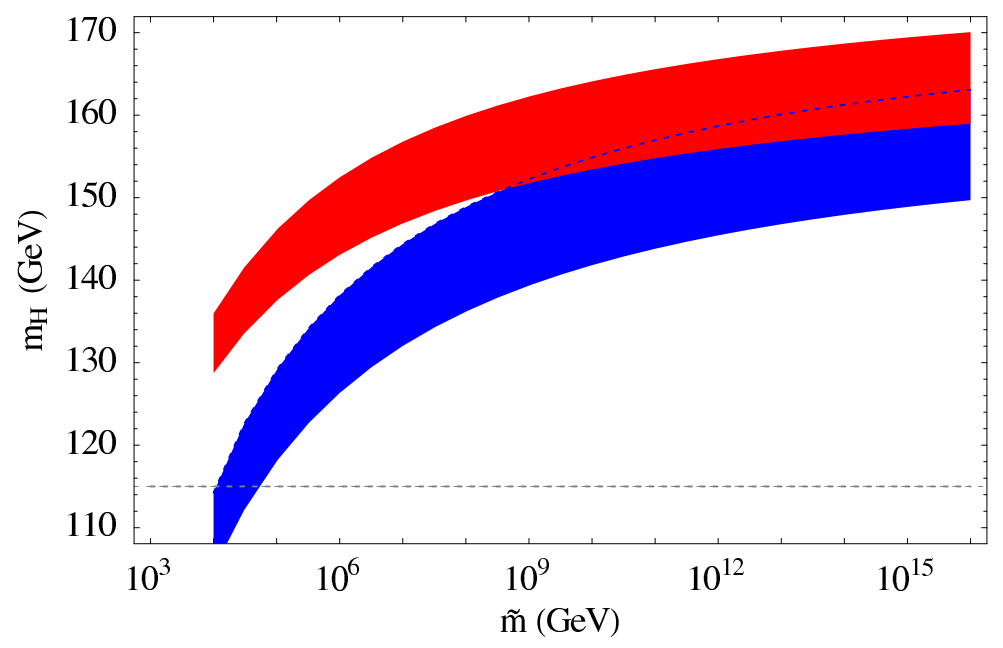

Figure 10: The value of the Higgs mass as a function of $\tilde{m}$. The bands include 1- $\sigma$ errors on $m_{t}$ and $\alpha_{s}\left(M_{Z}\right)$. The upper band corresponds to $\tan \beta=50$ and the lower one to $\tan \beta=1.5$.

\subsection{Dark Matter}

As discussed in the introduction, in the absence of the naturalness criterion, dark matter can provide the link between new physics and the electroweak scale. It is therefore crucial to study what are the implications of the request that the lightest neutralino is the dark matter particle. Differently than in ordinary low-energy supersymmetry, the parameter $\mu$ is not determined by electroweak symmetry breaking, but uniquely by the relic abundance calculation. In this section, we study this relation.

Let us first consider the case in which the lightest neutralino is mostly B-ino. Since squarks and sleptons are decoupled and the B-ino is a gauge singlet, its only interaction is through its coupling $\tilde{g}_{u, d}^{\prime}$ with Higgs and higgsinos, given in eq. (20). Therefore, if $\mu \gg M_{1}$, the B-ino is nearly decoupled and it annihilates too weakly in the early universe. This means that we need to consider values of $\mu$ comparable with $M_{1}$, and thus the lightest neutralino $\chi$ is always a mixture of gaugino and higgsino and never a pure state. Through the mixing, this state annihilates efficiently into Higgs and gauge bosons. The dominant contribution, when $\mu$ is not much larger than $M_{1}$ comes from $p$-wave annihilation into longitudinal gauge bosons which, for $M_{1} \gg M_{Z}$, gives a $\chi$ relic abundance

$$
\Omega_{\chi} h^{2} \simeq 0.1 \frac{\mu^{2}\left(M_{1}^{2}+\mu^{2}\right)^{2}}{m_{\chi}^{4} \mathrm{TeV}^{2}} .
$$

Now we turn to the case in which the lightest neutralino is mainly a higgsino. The higgsino has gauge interactions which survive in the limit $M_{1,2} \gg \mu$, and therefore it can be 
the dark matter, even in a pure state. Actually the coupling of the lightest higgsino with the $Z$ vanishes when $\mu \gg M_{Z}$. However, in this limit the other neutral and charged higgsinos are nearly degenerate in mass (and the lightest state is neutral) and off-diagonal couplings of the gauge bosons to higgsinos are allowed. When computing the relic abundance, it is therefore important to include the coannihilation of the various channels [19]. When this is done, we find that the relic abundance of a heavy higgsino in Split Supersymmetry is ${ }^{3}$

$$
\Omega_{\tilde{H}} h^{2}=0.09\left(\frac{\mu}{\mathrm{TeV}}\right)^{2}
$$

Using the 2- $\sigma$ range of dark matter density preferred after WMAP data [21]

$$
0.094<\Omega_{D M} h^{2}<0.129
$$

we find that a higgsino dark matter should have a mass in the range 1.0 to $1.2 \mathrm{TeV}$.

Next we consider a $W$-ino lightest neutralino. The $\mathrm{W}$-ino has also a neutral component that could play the rôle of the dark matter. It is not usually considered a standard candidate, because in ordinary low-energy supersymmetry with gaugino unification condition, it can never be the lightest state. However, the W-ino can become the LSP in anomaly mediation 22]. Both tree-level and one-loop effects contribute to make the neutral state belonging to the $S U(2)$ triplet lighter than the charged one [22]. In the limit of pure W-ino, the relic abundance in Split Supersymmetry is

$$
\Omega_{\tilde{W}} h^{2}=0.02\left(\frac{M_{2}}{\mathrm{TeV}}\right)^{2}
$$

Using eq. (36), we find that the mass range of a $\mathrm{W}$-ino dark matter is 2.0 to $2.5 \mathrm{TeV}$.

Let us now study the dependence of the neutralino relic abundance as we vary the parameters of Split Supersymmetry. The numerical result of the correlation between $\mu$ and $M_{2}$ is shown in fig. [1], assuming gaugino mass unification, $\tilde{m}=10^{5} \mathrm{GeV}$ and $\tan \beta=4$ and 20 . The ratio $M_{3} / M_{2}$ is determined by the equations discussed in sect. 3.3 .

For small values of $M_{2}$, the lightest neutralino is mainly $B$-ino, but as explained before, the higgsino component is non-negligible. When $M_{2}$ is larger than the gauge-boson masses, eq. (34) is a good approximation and therefore the correlation between $M_{2}$ and $\mu$ is roughly $\mu \propto M_{2}^{2 / 3}$, in agreement with the results shown in fig. 11] As $M_{2}$ grows, the gap between $\mu$ and $M_{1}$ gets reduces until we have the transition to the higgsino region, where the value

\footnotetext{
${ }^{3}$ The numerical calculations of the dark-matter relic abundances and detection rates presented in this paper have been performed using the fortran package DarkSUSY [20] adapted to the case of Split Supersymmetry.
} 

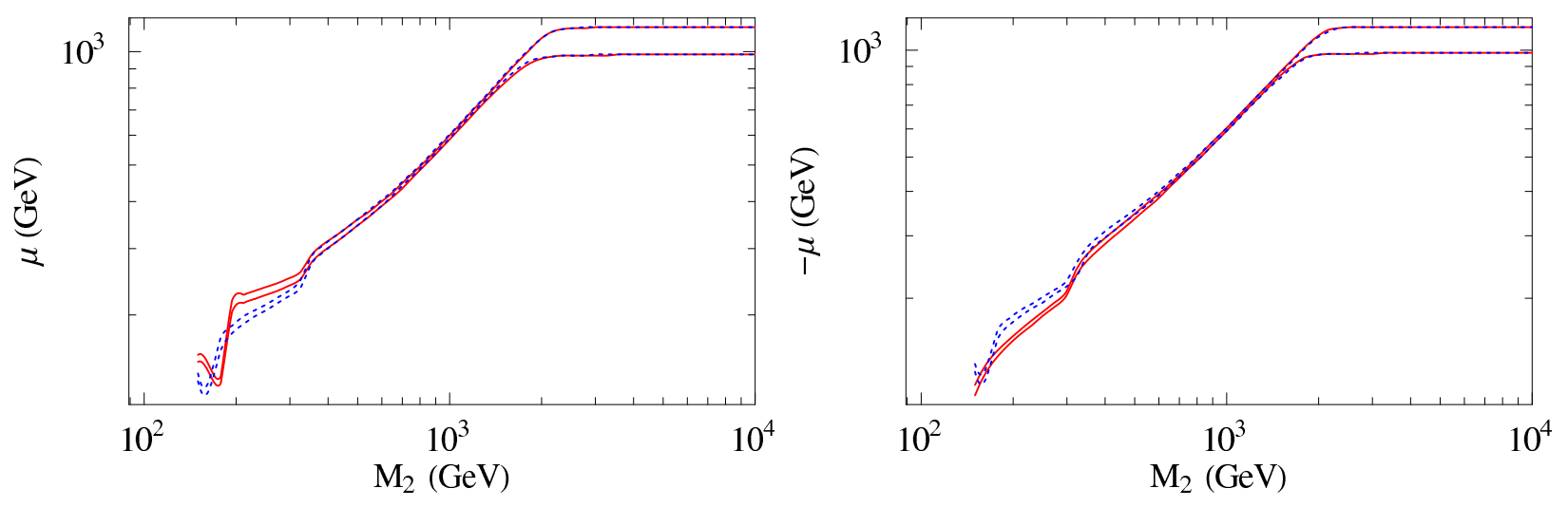

Figure 11: The bands give the values of $\mu$, as functions of $M_{2}$, consistent with the dark matter constraint $0.094<\Omega_{D M} h^{2}<0.129$, with gaugino mass unification The solid lines correspond to $\tan \beta=4$ and the dashed lines to $\tan \beta=20$. The left frame is for positive values of $\mu$ and the right frame for negative values of $\mu$.

of $\mu$ is uniquely determined by eq. (35). Thus the behaviour of the curves in fig. 11 is well described by the various limiting approximations.

Dark matter particles are actively searched for in underground experiments through their scatterings with nuclei. In the case of Split-Supersymmetry, since squarks and sleptons are very heavy, and the $Z-\chi^{0}-\chi^{0}$ coupling gives only a spin-dependent neutralino interaction with nuclei, the only contribution to the spin-independent cross section comes from the exchange of the Higgs boson 23]. To compare different experiments, it is customary to consider the neutralino scattering cross-section off a proton, which is given by

$$
\sigma_{p}=\left(N_{11} \tan \theta_{W}-N_{12}\right)^{2}\left(N_{13} \cos \beta-N_{14} \sin \beta\right)^{2}\left(\frac{115 \mathrm{GeV}}{m_{H}}\right)^{4} 4 \times 10^{-43} \mathrm{~cm}^{2} .
$$

Here $m_{H}$ is the Higgs mass and $N_{1, i}$ are the gaugino and higgsino components of the lightest neutralino, in standard notations. As shown by eq. (38), the $H-\chi^{0}-\chi^{0}$ coupling vanishes when the neutralino is a pure gaugino $\left(N_{13}=N_{14}=0\right)$ or pure higgsino $\left(N_{13}=N_{14}=0\right)$. Interestingly, Split Supersymmetry predicts that $\chi^{0}$ is a mixed state, as long as $M_{2}$ is not too large (see fig. 11). However, the scattering rate is rather small and it drops with the Higgs mass as $M_{H}^{-4}$. In fig. 12, we show the spin-independent neutralino-proton cross section for the same parameter choice as in fig. 11. We have normalized the cross section with a fixed value of $m_{H}$, to allow a simple scaling of the results. Notice how the detection rate is negligible when $\chi^{0}$ becomes higgsino $\left(M_{2} \gtrsim \mathrm{TeV}\right)$. Nevertheless, in the case of a mixed state and in a large range of the allowed Higgs mass, the signal is within the reach of future experiments, which will have a sensitivity up to $10^{-44}-10^{-45} \mathrm{~cm}^{2}$ for $m_{\chi}=1 \mathrm{TeV}$. 

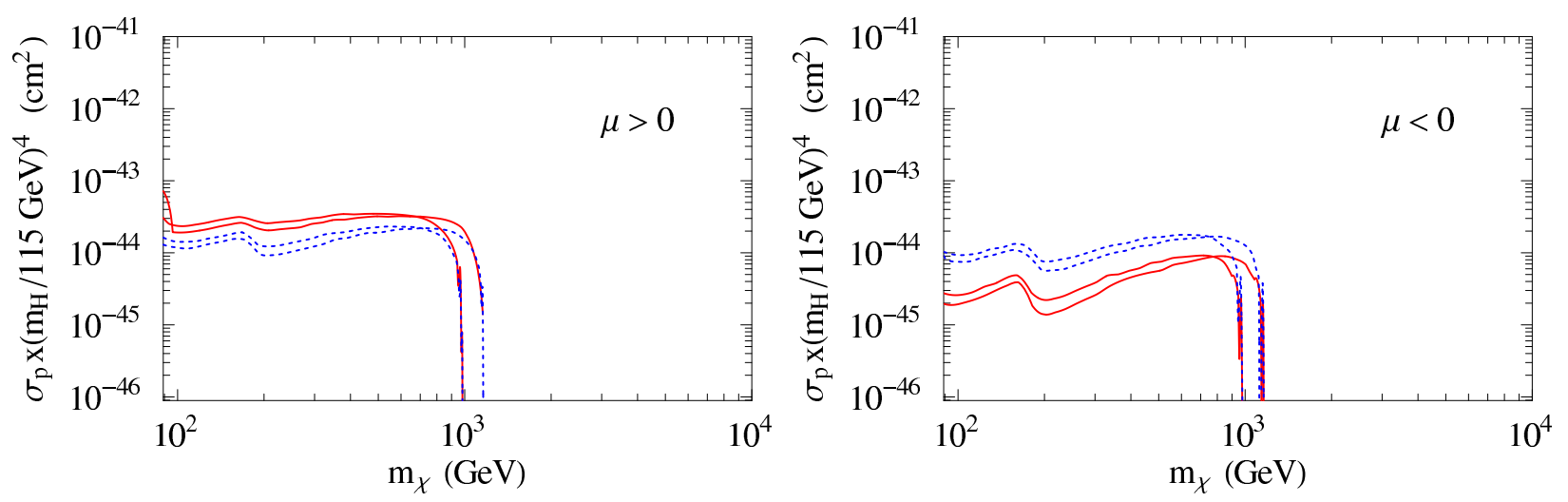

Figure 12: The bands give neutralino-proton cross section relevant for dark matter detection, as a function of the neutralino mass $m_{\chi}$. The cross section, which scales as $\sigma_{p} \propto m_{H}^{-4}$, has been calculated for $m_{H}=115 \mathrm{GeV}$. The parameters are such that the neutralino relic abundance is consistent with the dark matter constraint $0.094<\Omega_{D M} h^{2}<0.129$, assuming gaugino mass unification. The solid lines correspond to $\tan \beta=4$ and the dashed lines to $\tan \beta=20$. The left frame is for positive values of $\mu$ and the right frame for negative values of $\mu$.

\subsection{Collider Signals}

Our dark matter analysis has identified three preferred regions of Split Supersymmetry.

The first region is, for gaugino mass unification, when $M_{2}$ is below about $1-2 \mathrm{TeV}$, and the parameter $\mu$ is determined to be comparable (but slightly larger) than $M_{1}$. As $M_{2}$ grows, the degeneracy between $\mu$ and $M_{1}$ becomes more pronounced. The lightest neutralino is always a mixed state. In this region, the gaugino mass parameters can be as low as their present experimental lower limit, giving particularly interesting prospects for future searches.

The second case is when $\mu \simeq 1.0-1.2 \mathrm{TeV}$ and the gaugino masses are (arbitrarily) larger than $\mu$. This is, for instance, the situation when the $\mu$ parameter is radiatively generated, as considered in sect. 3.3. New particles can be considerably heavy.

Finally, there is the possibility that $M_{2} \simeq 2.0-2.5 \mathrm{TeV}$ and the other parameters are (arbitrarily) larger: this is the case with anomaly mediation. It is not a very pleasent scenario for collider experiments, since all new particles are very heavy. We want to stress however that, once we abandon the hierarchy problem, a lighter spectrum is not more natural than a heavy one. All solutions to the dark matter requirements are equally acceptable.

At the LHC, experiments can search for displaced vertices (if $\tilde{m}$ is low and the gluino decays inside the detector) or for stable coloured particles (for larger $\tilde{m}$ ) [10]. We expect that 
gluinos should be visible for masses up to about 2.5 TeV. This limits the reach on the parameter $M_{2}$ and certainly not all the region relevant for dark matter will be covered. Therefore, the search for neutralinos and charginos can be quite important. In Split Supersymmetry, neutralinos and charginos are produced at the LHC only through gauge-boson interaction, since there are no decay chains originated by gluino and squark decays. The trilepton channel is also not feasible, because of the small leptonic branching ratios of gauginos, in the limit of heavy sleptons.

There are two characteristics of Split Supersymmetry, which determine the collider phenomenology of neutralinos and charginos. (i) In the effective theory, the $B$-ino interacts only with Higgs and higgsinos, see eq. (20). Therefore, when $M_{1}$ is smaller than $M_{2}$ and $\mu$, we expect a significant probability of finding Higgs bosons in the final states of the decay chains of the heavy neutralinos and charginos. A complication exists when $M_{1}$ and $\mu$ become nearly mass degenerate. (ii) The phenomenology of Split Supersymmetry is different than the one of ordinary low-energy supersymmetry with heavy squarks, because of the value of the $\mu$ parameter. In the ordinary case, radiative electroweak breaking leads to a large $\mu$ and almost pure gauginos are the lightest states. In Split Supersymmetry $\mu$ is determined by dark matter considerations and higgsinos play an important rôle.

Identification of neutralinos and charginos at the LHC requires the analysis of signals with multi-Higgs and missing energy in the final state. Once the Higgs is discovered in the conventional channel and its mass determined, one can isolate multi- $\bar{b}-b$ pairs with the correct invariant mass from the background.

Linear colliders are most appropriate to extend the search for neutralinos and charginos. In particular, a multi-TeV collider like CLIC could entirely cover the cases of mixed-state or higgsino dark matter. The $W$-ino case requires center-of-mass energies up to $4-5 \mathrm{TeV}$. Moreover, a linear collider can determine the mass parameters and the gaugino couplings $\tilde{g}$, testing the relations of Split Supersymmetry.

Acknowledgments We wish to thank T. Jones, M. Mangano, T. Plehn, and R. Rattazzi for useful discussions.

Note added After completion of this paper, the paper in ref. [26] appeared, giving the renormalization-group equations for Split Supersymmetry at one-loop order. The equations for the gaugino coupling $\tilde{g}_{u}$, for the weak-gaugino mass, and for the Higgs coupling do not agree with the results given in our appendix. Our results correctly match the supersymmetric case. 


\section{Appendix}

In this appendix we give the renormalization-group equations for Split Supersymmetry. They have been derived from the general expressions given in ref. 24]. We have checked that when the contribution from heavy scalars is included, our equations reproduce the supersymmetric result, given in ref. [25].

The 2-loop renormalization-group equation for the gauge couplings is

$$
\begin{aligned}
(4 \pi)^{2} \frac{d}{d t} g_{i}=g_{i}^{3} b_{i} & +\frac{g_{i}^{3}}{(4 \pi)^{2}}\left[\sum_{j=1}^{3} B_{i j} g_{j}^{2}-\sum_{\alpha=u, d, e} d_{i}^{\alpha} \operatorname{Tr}\left(h^{\alpha \dagger} h^{\alpha}\right)\right. \\
& \left.-d_{W}\left(\tilde{g}_{u}^{2}+\tilde{g}_{d}^{2}\right)-d_{B}\left(\tilde{g}_{u}^{\prime 2}+\tilde{g}_{d}^{\prime 2}\right)\right]
\end{aligned}
$$

where $t=\ln \bar{\mu}$ and $\bar{\mu}$ is the renormalization scale. We use the convention $g_{1}^{2}=(5 / 3) g^{\prime 2}$. Equation (39) is scheme-independent up to the two-loop order.

In the effective theory below $\tilde{m}$, the $\beta$-function coefficients are

$$
\begin{aligned}
& b=\left(\frac{9}{2},-\frac{7}{6},-5\right) \quad B=\left(\begin{array}{ccc}
\frac{104}{25} & \frac{18}{5} & \frac{44}{5} \\
\frac{6}{5} & \frac{106}{3} & 12 \\
\frac{11}{10} & \frac{9}{2} & 22
\end{array}\right) \\
& d^{u}=\left(\frac{17}{10}, \frac{3}{2}, 2\right) \quad d^{d}=\left(\frac{1}{2}, \frac{3}{2}, 2\right) \quad d^{e}=\left(\frac{3}{2}, \frac{1}{2}, 0\right) \\
& d^{W}=\left(\frac{9}{20}, \frac{11}{4}, 0\right) \quad d^{B}=\left(\frac{3}{20}, \frac{1}{4}, 0\right) .
\end{aligned}
$$

Above the scale $\tilde{m}$, we recover the supersymmetric result (identifying $h^{\alpha}$ with $\lambda^{\alpha *}$ )

$$
\begin{aligned}
& b=\left(\frac{33}{5}, 1,-3\right) \quad B=\left(\begin{array}{ccc}
\frac{199}{25} & \frac{27}{5} & \frac{88}{5} \\
\frac{9}{5} & 25 & 24 \\
\frac{11}{5} & 9 & 14
\end{array}\right) \\
& d^{u}=\left(\frac{26}{5}, 6,4\right) \quad d^{d}=\left(\frac{14}{5}, 6,4\right) \quad d^{e}=\left(\frac{18}{5}, 2,0\right) \quad d^{W}=d^{B}=0 .
\end{aligned}
$$

Below the mass of the lightest neutralino, the theory coincides with the SM with one Higgs doublet and

$$
\begin{aligned}
& b=\left(\frac{41}{10},-\frac{19}{6},-7\right) \quad B=\left(\begin{array}{ccc}
\frac{199}{50} & \frac{27}{10} & \frac{44}{5} \\
\frac{9}{10} & \frac{35}{6} & 12 \\
\frac{11}{10} & \frac{9}{2} & -26
\end{array}\right) \\
& d^{u}=\left(\frac{17}{10}, \frac{3}{2}, 2\right) \quad d^{d}=\left(\frac{1}{2}, \frac{3}{2}, 2\right) \quad d^{e}=\left(\frac{3}{2}, \frac{1}{2}, 0\right) \quad d^{W}=d^{B}=0 .
\end{aligned}
$$


Since Yukawa and gaugino couplings appear in eq. (39) only in the two-loop part, we need their evolution up to the one-loop order. For the Yukawa couplings, we find

$$
\begin{aligned}
(4 \pi)^{2} \frac{d}{d t} h^{u} & =h^{u}\left(-3 \sum_{i=1}^{3} c_{i}^{u} g_{i}^{2}+\frac{3}{2} h^{u \dagger} h^{u}-\frac{3}{2} h^{d \dagger} h^{d}+T\right) \\
(4 \pi)^{2} \frac{d}{d t} h^{d} & =h^{d}\left(-3 \sum_{i=1}^{3} c_{i}^{d} g_{i}^{2}-\frac{3}{2} h^{u \dagger} h^{u}+\frac{3}{2} h^{d^{\dagger}} h^{d}+T\right) \\
(4 \pi)^{2} \frac{d}{d t} h^{e} & =h^{e}\left(-3 \sum_{i=1}^{3} c_{i}^{e} g_{i}^{2}+\frac{3}{2} h^{e \dagger} h^{e}+T\right) .
\end{aligned}
$$

Below the scale $\tilde{m}$, we find

$$
\begin{gathered}
T=\operatorname{Tr}\left(3 h^{u \dagger} h^{u}+3 h^{d \dagger} h^{d}+h^{e \dagger} h^{e}\right)+\frac{3}{2}\left(\tilde{g}_{u}^{2}+\tilde{g}_{d}^{2}\right)+\frac{1}{2}\left(\tilde{g}_{u}^{\prime 2}+\tilde{g}_{d}^{\prime 2}\right) \\
c^{u}=\left(\frac{17}{60}, \frac{3}{4}, \frac{8}{3}\right) \quad c^{d}=\left(\frac{1}{12}, \frac{3}{4}, \frac{8}{3}\right) \quad c^{e}=\left(\frac{3}{4}, \frac{3}{4}, 0\right) .
\end{gathered}
$$

This result is valid also for the SM, if we take $T=\operatorname{Tr}\left(3 h^{u \dagger} h^{u}+3 h^{d \dagger} h^{d}+h^{{ }^{\dagger}} h^{e}\right)$.

Above the scale $\tilde{m}$, the renormalization-group equations of the Yukawa couplings are

$$
\begin{aligned}
(4 \pi)^{2} \frac{d}{d t} \lambda^{u} & =\lambda^{u}\left[-2 \sum_{i=1}^{3} c_{i}^{u} g_{i}^{2}+3 \lambda^{u \dagger} \lambda^{u}+\lambda^{d \dagger} \lambda^{d}+3 \operatorname{Tr}\left(\lambda^{u \dagger} \lambda^{u}\right)\right] \\
(4 \pi)^{2} \frac{d}{d t} \lambda^{d} & =\lambda^{d}\left[-2 \sum_{i=1}^{3} c_{i}^{d} g_{i}^{2}+\lambda^{u \dagger} \lambda^{u}+3 \lambda^{d \dagger} \lambda^{d}+\operatorname{Tr}\left(3 \lambda^{d \dagger} \lambda^{d}+\lambda^{e \dagger} \lambda^{e}\right)\right] \\
(4 \pi)^{2} \frac{d}{d t} \lambda^{e} & =\lambda^{e}\left[-2 \sum_{i=1}^{3} c_{i}^{e} g_{i}^{2}+3 \lambda^{e \dagger} \lambda^{e}+\operatorname{Tr}\left(3 \lambda^{d \dagger} \lambda^{d}+\lambda^{e \dagger} \lambda^{e}\right)\right] . \\
c^{u} & =\left(\frac{13}{30}, \frac{3}{2}, \frac{8}{3}\right) \quad c^{d}=\left(\frac{7}{30}, \frac{3}{2}, \frac{8}{3}\right) \quad c^{e}=\left(\frac{9}{10}, \frac{3}{2}, 0\right) .
\end{aligned}
$$

The renormalization-group equations for the gaugino couplings defined in eq. (20) are

$$
\begin{gathered}
(4 \pi)^{2} \frac{d}{d t} \tilde{g}_{u}=-3 \tilde{g}_{u} \sum_{i=1}^{3} C_{i} g_{i}^{2}+\frac{5}{4} \tilde{g}_{u}^{3}-\frac{1}{2} \tilde{g}_{u} \tilde{g}_{d}^{2}+\frac{1}{4} \tilde{g}_{u} \tilde{g}_{u}^{\prime 2}+\tilde{g}_{d} \tilde{g}_{d}^{\prime} \tilde{g}_{u}^{\prime}+\tilde{g}_{u} T \\
(4 \pi)^{2} \frac{d}{d t} \tilde{g}_{u}^{\prime}=-3 \tilde{g}_{u}^{\prime} \sum_{i=1}^{3} C_{i}^{\prime} g_{i}^{2}+\frac{3}{4} \tilde{g}_{u}^{\prime 3}+\frac{3}{2} \tilde{g}_{u}^{\prime} \tilde{g}_{d}^{\prime 2}+\frac{3}{4} \tilde{g}_{u}^{\prime} \tilde{g}_{u}^{2}+3 \tilde{g}_{d}^{\prime} \tilde{g}_{d} \tilde{g}_{u}+\tilde{g}_{u}^{\prime} T \\
(4 \pi)^{2} \frac{d}{d t} \tilde{g}_{d}=-3 \tilde{g}_{d} \sum_{i=1}^{3} C_{i} g_{i}^{2}+\frac{5}{4} \tilde{g}_{d}^{3}-\frac{1}{2} \tilde{g}_{d} \tilde{g}_{u}^{2}+\frac{1}{4} \tilde{g}_{d} \tilde{g}_{d}^{\prime 2}+\tilde{g}_{u} \tilde{g}_{u}^{\prime} \tilde{g}_{d}^{\prime}+\tilde{g}_{d} T \\
(4 \pi)^{2} \frac{d}{d t} \tilde{g}_{d}^{\prime}=-3 \tilde{g}_{d}^{\prime} \sum_{i=1}^{3} C_{i}^{\prime} g_{i}^{2}+\frac{3}{4} \tilde{g}_{d}^{\prime 3}+\frac{3}{2} \tilde{g}_{d}^{\prime} \tilde{g}_{u}^{\prime 2}+\frac{3}{4} \tilde{g}_{d}^{\prime} \tilde{g}_{d}^{2}+3 \tilde{g}_{u}^{\prime} \tilde{g}_{u} \tilde{g}_{d}+\tilde{g}_{d}^{\prime} T, \\
C=\left(\frac{3}{20}, \frac{11}{4}, 0\right) \quad C^{\prime}=\left(\frac{3}{20}, \frac{3}{4}, 0\right) .
\end{gathered}
$$


The renormalization-group equations for the gaugino masses and the $\mu$ parameter below the scale $\tilde{m}$ are

$$
\begin{aligned}
(4 \pi)^{2} \frac{d}{d t} M_{3} & =-18 g_{3}^{2} M_{3}\left(1+\frac{c_{\tilde{g}} g_{3}^{2}}{(4 \pi)^{2}}\right) \\
(4 \pi)^{2} \frac{d}{d t} M_{2} & =\left(-12 g_{2}^{2}+\tilde{g}_{u}^{2}+\tilde{g}_{d}^{2}\right) M_{2}+4 \tilde{g}_{u} \tilde{g}_{d} \mu \\
(4 \pi)^{2} \frac{d}{d t} M_{1} & =\left(\tilde{g}_{u}^{\prime 2}+\tilde{g}_{d}^{\prime 2}\right) M_{1}+4 \tilde{g}_{u}^{\prime} \tilde{g}_{d}^{\prime} \mu \\
(4 \pi)^{2} \frac{d}{d t} \mu & =\frac{1}{4}\left[-18\left(\frac{g_{1}^{2}}{5}+g_{2}^{2}\right)+3\left(\tilde{g}_{u}^{2}+\tilde{g}_{d}^{2}\right)+\tilde{g}_{u}^{\prime 2}+\tilde{g}_{d}^{\prime 2}\right] \mu+3 \tilde{g}_{u} \tilde{g}_{d} M_{2}+\tilde{g}_{u}^{\prime} \tilde{g}_{d}^{\prime} M_{1}(64
\end{aligned}
$$

For $M_{3}$ we have included also the next-to-leading order correction and we find $c_{\tilde{g}}=38 / 3$ in $\overline{\mathrm{MS}}$ and $c_{\tilde{g}}=10 \mathrm{in} \overline{\mathrm{DR}}$. The relation between the gluino running and pole mass is

$$
M_{\tilde{g}}^{\text {pole }}=M_{3}(\bar{\mu})\left[1+\frac{g_{3}^{2}}{(4 \pi)^{2}}\left(C_{\tilde{g}}+9 \ln \frac{\bar{\mu}^{2}}{M_{3}^{2}}\right)\right],
$$

with $C_{\tilde{g}}=12$ in $\overline{\mathrm{MS}}$ and $c_{\tilde{g}}=15$ in $\overline{\mathrm{DR}}$.

Above the scale $\tilde{m}$, the equations for the gaugino masses and $\mu$ are given by

$$
\begin{aligned}
(4 \pi)^{2} \frac{d}{d t} M_{i} & =2 b_{i} g_{i}^{2} M_{i} \\
(4 \pi)^{2} \frac{d}{d t} \mu & =\left[-3 g_{2}^{2}-\frac{3}{5} g_{1}^{2}+\operatorname{Tr}\left(3 \lambda^{u \dagger} \lambda^{u}+3 \lambda^{d \dagger} \lambda^{d}+\lambda^{e \dagger} \lambda^{e}\right)\right] \mu .
\end{aligned}
$$

The two-loop expression of the gluino mass can be found in ref. [25]. In our numerical analysis we have set to zero the trilinear $A$-terms.

Finally, the equation for the Higgs quartic coupling is

$$
\begin{aligned}
(4 \pi)^{2} \frac{d}{d t} \lambda= & 12 \lambda^{2}+\lambda\left[-9\left(\frac{g_{1}^{2}}{5}+g_{2}^{2}\right)+6\left(\tilde{g}_{u}^{2}+\tilde{g}_{d}^{2}\right)+2\left(\tilde{g}_{u}^{\prime 2}+\tilde{g}_{d}^{\prime 2}\right)\right. \\
& \left.+4 \operatorname{Tr}\left(3 h^{u \dagger} h^{u}+3 h^{d \dagger} h^{d}+h^{e \dagger} h^{e}\right)\right]+\frac{9}{2}\left(\frac{g_{2}^{4}}{2}+\frac{3 g_{1}^{4}}{50}+\frac{g_{1}^{2} g_{2}^{2}}{5}\right) \\
& -5\left(\tilde{g}_{u}^{4}+\tilde{g}_{d}^{4}\right)-2 \tilde{g}_{u}^{2} \tilde{g}_{d}^{2}-\left(\tilde{g}_{u}^{\prime 2}+\tilde{g}_{d}^{\prime 2}\right)^{2}-2\left(\tilde{g}_{u} \tilde{g}_{u}^{\prime}+\tilde{g}_{d} \tilde{g}_{d}^{\prime}\right)^{2} \\
& -4 \operatorname{Tr}\left[3\left(h^{u \dagger} h^{u}\right)^{2}+3\left(h^{d \dagger} h^{d}\right)^{2}+\left(h^{e \dagger} h^{e}\right)^{2}\right] .
\end{aligned}
$$

\section{References}

[1] A. Vilenkin, Phys. Rev. Lett. 74 (1995) 846 [arXiv gr-qc/9406010; S. Weinberg, Phys. Rev. Lett. 59 (1987) 2607. 
[2] R. Bousso and J. Polchinski, JHEP 0006, 006 (2000) [arXiv/hep-th/0004134; S. Kachru, R. Kallosh, A. Linde and S. P. Trivedi, Phys. Rev. D 68, 046005 (2003) [arXiv hep-th/0301240]; L. Susskind, arXiv hep-th/0302219 F. Denef and M. R. Douglas, arXiv:hep-th/0404116.

[3] N. Arkani-Hamed and S. Dimopoulos, arXiv hep-th/0405159.

[4] G. Altarelli and M. W. Grunewald, arXiv:hep-ph/0404165.

[5] S. Aoki et al. [JLQCD Collaboration], Phys. Rev. D 62, 014506 (2000) [arXiv hep-lat/9911026].

[6] Y. Suzuki et al. [TITAND Working Group Collaboration], arXiv hep-ex/0110005.

[7] D. Choudhury, T. M. P. Tait and C. E. M. Wagner, Phys. Rev. D 65, 053002 (2002) [arXiv hep-ph/0109097]; D. E. Morrissey and C. E. M. Wagner, Phys. Rev. D 69, 053001 (2004) [arXiv hep-ph/0308001].

[8] L. E. Ibanez, Phys. Lett. B 126 (1983) 196 [Erratum-ibid. B 130 (1983) 463]; J. E. Bjorkman and D. R. T. Jones, Nucl. Phys. B 259 (1985) 533; T. G. Rizzo, Phys. Rev. D 45, 3903 (1992).

[9] G. F. Giudice and A. Masiero, Phys. Lett. B 206, 480 (1988).

[10] H. Baer, K. m. Cheung and J. F. Gunion, Phys. Rev. D 59, 075002 (1999) [arXiv hep-ph/9806361.

[11] P. F. Smith, J. R. J. Bennett, G. J. Homer, J. D. Lewin, H. E. Walford and W. A. Smith, Nucl. Phys. B 206, 333 (1982).

[12] T. K. Hemmick et al., Phys. Rev. D 41, 2074 (1990).

[13] L. J. Hall, Nucl. Phys. B 178, 75 (1981).

[14] L. J. Hall, R. Rattazzi and U. Sarid, Phys. Rev. D 50, 7048 (1994) [arXiv hep-ph/9306309.

[15] F. Vissani and A. Y. Smirnov, Phys. Lett. B 341 (1994) 173 [arXiv/hep-ph/9405399; A. Brignole, H. Murayama and R. Rattazzi, Phys. Lett. B 335 (1994) 345 [arXiv:hep-ph/9406397]. 
[16] H. C. Cheng, J. L. Feng and N. Polonsky, Phys. Rev. D 56, 6875 (1997) [arXiv hep-ph/9706438; E. Katz, L. Randall and S. f. Su, Nucl. Phys. B 536 (1998) 3 [arXiv hep-ph/9801416; S. Kiyoura, M. M. Nojiri, D. M. Pierce and Y. Yamada, Phys. Rev. D 58 (1998) 075002 [arXiv hep-ph/9803210].

[17] L. Randall and R. Sundrum, Nucl. Phys. B 557, 79 (1999) [arXiv hep-th/9810155]; G. F. Giudice, M. A. Luty, H. Murayama and R. Rattazzi, JHEP 9812, 027 (1998) [arXiv:hep-ph/9810442].

[18] R. Barate et al., Phys. Lett. B 565 (2003) 61 [arXiv:hep-ex/0306033].

[19] J. Edsjo and P. Gondolo, Phys. Rev. D 56 (1997) 1879 [arXiv hep-ph/9704361].

[20] P. Gondolo, J. Edsjo, P. Ullio, L. Bergstrom, M. Schelke and E. A. Baltz, arXivastro-ph/0211238

[21] C. L. Bennett et al., Astrophys. J. Suppl. 148 (2003) 1 [arXiv astro-ph/0302207]; D. N. Spergel et al., Astrophys. J. Suppl. 148 (2003) 175 [arXiv astro-ph/0302209].

[22] T. Gherghetta, G. F. Giudice and J. D. Wells, Nucl. Phys. B 559, 27 (1999) [arXiv hep-ph/9904378].

[23] R. Barbieri, M. Frigeni and G. F. Giudice, Nucl. Phys. B 313, 725 (1989).

[24] M. E. Machacek and M. T. Vaughn, Nucl. Phys. B 222, 83 (1983); Nucl. Phys. B 236, 221 (1984); Nucl. Phys. B 249, 70 (1985).

[25] S. P. Martin and M. T. Vaughn, Phys. Rev. D 50, 2282 (1994) [arXiv hep-ph/9311340].

[26] A. Arvanitaki, C. Davis, P. W. Graham and J. G. Wacker, arXiv hep-ph/0406034. 